\title{
Review Article \\ Prospects for Studies of the Free Fall and Gravitational Quantum States of Antimatter
}

\author{
G. Dufour, ${ }^{1}$ D. B. Cassidy, ${ }^{2}$ P. Crivelli, ${ }^{3}$ P. Debu, ${ }^{4}$ A. Lambrecht, ${ }^{1}$ \\ V. V. Nesvizhevsky, ${ }^{5}$ S. Reynaud, ${ }^{1}$ A. Yu. Voronin, ${ }^{6}$ and T. E. Wall ${ }^{2}$ \\ ${ }^{1}$ Laboratoire Kastler-Brossel, CNRS, ENS, Collège de France, UPMC, Campus Jussieu, 75252 Paris, France \\ ${ }^{2}$ Department of Physics and Astronomy, University College London, Gower Street, London WC1E 6BT, UK \\ ${ }^{3}$ ETH Zurich, 8093 Zurich, Switzerland \\ ${ }^{4}$ Institut de Recherche sur les lois Fondamentales de l'Univers, CEA Saclay, 91191 Gif-sur-Yvette, France \\ ${ }^{5}$ Institut Max von Laue-Paul Langevin, 6 rue Jules Horowitz, 38042 Grenoble, France \\ ${ }^{6}$ P.N. Lebedev Physical Institute, 53 Leninsky Prospekt, 117924 Moscow, Russia
}

Correspondence should be addressed to G. Dufour; gabriel.dufour@upmc.fr

Received 29 July 2014; Accepted 1 October 2014

Academic Editor: Ignatios Antoniadis

Copyright (C) 2015 G. Dufour et al. This is an open access article distributed under the Creative Commons Attribution License, which permits unrestricted use, distribution, and reproduction in any medium, provided the original work is properly cited. The publication of this article was funded by SCOAP . $^{3}$

\begin{abstract}
Different experiments are ongoing to measure the effect of gravity on cold neutral antimatter atoms such as positronium, muonium, and antihydrogen. Among those, the project GBAR at CERN aims to measure precisely the gravitational fall of ultracold antihydrogen atoms. In the ultracold regime, the interaction of antihydrogen atoms with a surface is governed by the phenomenon of quantum reflection which results in bouncing of antihydrogen atoms on matter surfaces. This allows the application of a filtering scheme to increase the precision of the free fall measurement. In the ultimate limit of smallest vertical velocities, antihydrogen atoms are settled in gravitational quantum states in close analogy to ultracold neutrons (UCNs). Positronium is another neutral system involving antimatter for which free fall under gravity is currently being investigated at UCL. Building on the experimental techniques under development for the free fall measurement, gravitational quantum states could also be observed in positronium. In this contribution, we report on the status of the ongoing experiments and discuss the prospects of observing gravitational quantum states of antimatter and their implications.
\end{abstract}

\section{Introduction}

At present, together with the dark matter problem, one of the most tantalizing open questions in physics is the baryonantibaryon asymmetry; that is, why are we living in a matterdominated universe? Where did all the antimatter go? Different theoretical and experimental efforts trying to address this question are ongoing, including activities focusing on the gravitational behavior of antimatter [1-5]. No compelling theoretical argument seems to support that a difference between the gravitational behavior of matter and antimatter should be expected [6], although some attempts have been made to show the contrary [7-11]. Moreover observations and experiments have been interpreted as evidence against the existence of "antigravity" type forces [12-15]. However, those could be argued to be model dependent and, therefore, a simple free fall measurement is preferable. This justifies ongoing experimental efforts in that direction. A first attempt in this direction was made recently by the ALPHA collaboration [1] that bounds the ratio of gravitational mass to inertial mass of antihydrogen between -65 and 110 .

The idea of directly measuring the gravitational force acting on antiparticles in the Earth's field goes back many decades, from the work of Witteborn and Fairbank, attempting to measure the acceleration of gravity for electrons and, eventually, positrons [16] to the PS200 experiment at CERN in the 1980s which included measurements on antiprotons [17]. Such measurements are extremely difficult because 
measuring the force of gravity on a charged particle requires a physically unrealistic elimination of stray electromagnetic fields. The obvious solution to this problem is to use neutral antimatter particles. However, at the present time, it is not technically feasible to do so; antineutrons cannot be produced in a controllable manner and antineutrinos are similarly elusive to experimenters. One may instead consider using composite systems that are electrically neutral, in which case it is only necessary to contend with dipole moments. Only a few systems that are composed of or contain some fraction of antimatter are available for scientific study. These are antihydrogen, muonium, and positronium, which have all been suggested as possible candidates for gravity measurements [18].

Selecting between different experimental methods, one should aim at precision experiments as they are much more strongly motivated theoretically. Among these, the method of quantum gravitational spectroscopy stands out by its remarkable statistical sensitivity and its cleanness from a systematic point of view.

Gravitational quantum states are solutions of the Schrödinger equation in a gravity field above a surface. They are characterized by the following energy $\left(E_{n}\right)$ and spatial scale $\left(H_{n}\right)$ :

$$
\begin{gathered}
E_{n}=\varepsilon_{0} \lambda_{n}, \quad \varepsilon_{0}=\sqrt[3]{\frac{\hbar^{2} M^{2} g^{2}}{2 m}}, \\
H_{n}=\frac{E_{n}}{M g}, \\
\operatorname{Ai}\left(-\lambda_{n}\right)=0, \\
\lambda_{n} \approx\{2.34,4.09,5.52,6.79,7.94,9.02,10.04, \ldots\} .
\end{gathered}
$$

Here, $M$ is the gravitational mass of the particle, $m$ is its inertial mass (we distinguish between $M$ and $m$ in view of discussing EP tests), $g$ is the gravitational field intensity near the Earth's surface, $\bar{g}=\mathrm{Mg} / \mathrm{m}$ is the acceleration of the particle in that field, and $\operatorname{Ai}(x)$ is the Airy function $[19,20]$. For neutrons and antihydrogen atoms, the height of the lowest gravitational level is $13.7 \mu \mathrm{m}$. For positronium, whose mass is approximately 1000 times smaller, it extends over $1.3 \mathrm{~mm}$. The frequency of transitions between first and second quantum states equals $254 \mathrm{~Hz}$ for neutrons and antihydrogen and $26 \mathrm{~Hz}$ for positronium. The corresponding characteristic times needed to form quantum states are $0.5 \mathrm{~ms}$ and $5 \mathrm{~ms}$, respectively.

Quantum gravitational states were observed for the first time with neutrons by measuring their transmission through a slit made of a mirror and an absorber in the GRANIT experiment [21]. If the distance between the mirror and the absorber (which is a rough surface used as a scatterer to mix the velocity components) is much higher than the turning point for the corresponding gravitational quantum state, the neutrons pass through the slit without significant losses. As the slit size decreases, the absorber starts approaching the size of the neutron wave function and the probability of neutron loss increases. If the slit size is smaller than the characteristic size of the neutron wave function in the lowest quantum state, the slit is not transparent for neutrons as was demonstrated experimentally.

Here, we analyze, in detail, several experiments which will study the free fall of antiatoms and argue that the observation of gravitational quantum states of antimatter is feasible. In Section 2, we describe the forthcoming $\overline{\mathrm{H}}$ experiment GBAR. We explain in Section 3 the quantum reflection mechanism which allows the formation of gravitational quantum states of $\overline{\mathrm{H}}$ above material surfaces. In Section 4 , we show how the filtering scheme of the GRANIT experiment could be implemented in GBAR and, in Section 5, we describe a possible spectroscopy of gravitational quantum states of $\overline{\mathrm{H}}$. Section 6 reviews the status of positronium free fall experiment at UCL and Section 7 explores the possibility of observing gravitational quantum states of positronium.

\section{The GBAR Experiment}

GBAR is an experiment in preparation at CERN. Its goal is to measure the gravitational acceleration $(\bar{g}=M g / m)$ imparted to freely falling antihydrogen atoms, in order to perform a direct experimental test of the Weak Equivalence Principle with antimatter. The objective is to reach a relative precision on $\bar{g}$ of $1 \%$ in a first stage, with the perspective to reach a much higher precision using quantum gravitational states in a second stage, as is described in Section 5.

The principle of the experiment is described in detail in [22] and is briefly recalled here. It is based on an idea proposed in [23]. Antihydrogen ions $\overline{\mathrm{H}}^{+}$are produced, trapped, and sympathetically cooled to around $10 \mu \mathrm{K}$. The excess positron is detached by a laser pulse, which gives the start signal for the free fall of the ultracold antihydrogen atom $\overline{\mathrm{H}}$. The $\overline{\mathrm{H}}$ subsequent annihilation on a plate is detected and provides the information to measure $\bar{g}$. The choice of producing $\overline{\mathrm{H}}^{+}$ions to get ultracold antihydrogen atom is the specificity of the GBAR experiment. It is very costly in statistics but makes the cooling to $\mu \mathrm{K}$ temperatures a realistic aim.

We report, in this section, on three recent progresses in the preparation of the experiment: estimations of the $\overline{\mathrm{H}}^{+}$ production cross-sections, accumulation of positrons, and cooling of the $\overline{\mathrm{H}}^{+}$ions.

2.1. Production Cross-Sections of $\overline{\mathrm{H}}^{+}$Ions. The $\overline{\mathrm{H}}$ production proceeds in two steps: $\bar{p}+\mathrm{Ps} \rightarrow \overline{\mathrm{H}}+\mathrm{e}^{-}$(1) followed by $\overline{\mathrm{H}}+\mathrm{Ps} \rightarrow \overline{\mathrm{H}}^{+}+\mathrm{e}^{-}$(2). The Ps symbol stands for positronium. The cross-sections of these reactions are not well known and are very low. The matter counterpart of the first one has been measured. It is around $10^{-15} \mathrm{~cm}^{2}\left(10^{9}\right.$ barn $)$ for tens of $\mathrm{keV}$ protons [24]. The second one is estimated to be around $10^{-16} \mathrm{~cm}^{2}\left(10^{8}\right.$ barn) [25].

New calculations of these reactions have been performed in which the first excitations levels for the Ps (up to $n=3$ ) and the $\overline{\mathrm{H}}$ (up to $n=5$ ) have been considered. The results suggest that the production of $\overline{\mathrm{H}}^{+}$can be efficiently enhanced by using either a fraction of $\operatorname{Ps}(2 \mathrm{p})$ and a $2 \mathrm{keV}$ antiproton beam or a fraction $\mathrm{Ps}(3 \mathrm{~d})$ and antiprotons with kinetic energy below $1 \mathrm{keV}$ [26]. The product of the cross-sections of reactions 
(1) and (2) reaches values around $10^{-29} \mathrm{~cm}^{4}\left(10^{19} \mathrm{barn}^{2}\right)$ for an optimized fraction of excited Ps. Simulations are underway to estimate the effective gain with a realistic experimental setup.

This shows that very low energy antiprotons are needed. The extremely low energy antiproton (ELENA) ring which is in construction at CERN and which will complement the antiproton decelerator (AD) will provide $75 \mathrm{~ns}$ rms bunches of $5 \times 10^{6} 100 \mathrm{keV}$ antiprotons every $100 \mathrm{~s}$. Those have to be further decelerated and cooled to match the GBAR requirements. The decelerator is under construction at CSNSM (Centre de Sciences Nucléaires et de Sciences de la Matière) in Orsay, France.

\subsection{Positron Accumulation. In addition to a high flux of low} energy antiprotons, the production of $\overline{\mathrm{H}}^{+}$via reactions (1) and (2) requires to form a dense cloud of positronium. It has been shown that Ps can be efficiently produced by dumping few $\mathrm{keV}$ positrons on mesoporous silica films. Yields of 30 to $40 \%$ depending on the incident positron energy (few keV) have been measured $[27,28]$. The accumulation of a very large number of positrons, around $2 \times 10^{10}$, between two ejections of antiprotons from ELENA is thus necessary to produce a dense enough positronium cloud.

A demonstration facility for the production and accumulation of positrons is currently running at CEA/Saclay. It consists of a low energy electron linear accelerator (LINAC), a high field Penning-Malmberg trap from the Atomic Physics Laboratory in RIKEN, Japan, and a dedicated beam line for further studies of positron-positronium conversion and for applications in material science. In addition, a laser system is now being built at LKB (Laboratoire Kastler-Brossel) in Paris to test the excitation of the positronium which will be formed downstream of the trap.

The LINAC produces a $4.3 \mathrm{MeV}$ electron bunched beam. The bunch length is $200 \mu \mathrm{s}$, and the LINAC runs at $200 \mathrm{~Hz}$, producing a mean current of $120 \mu \mathrm{A}$. Electrons are sent onto a tungsten mesh moderator. A flux of typically $3 \times 10^{6}$ slow (few $100 \mathrm{eV}$ ) positrons per second is driven towards the Penning trap through a vacuum tube equipped with solenoid coils producing a $80 \mathrm{mT}$ field. They are accelerated to around $1 \mathrm{keV}$ to enter the high magnetic field $(5 \mathrm{~T})$ region. They reach the Penning trap which is made of 23 cylindrical electrodes, surrounded by 4 additional long electrodes to control the admission and the trapping of incident particles. Positrons make a round trip in less than $100 \mathrm{~ns}$. In order to trap them, it is necessary to compress the $200 \mu$ s bunch. This is done by applying a varying voltage (20 to $150 \mathrm{~V}$ ) when extracting the slow positron from the moderator. In this way, it is possible to close the entrance of the trap before the bunch escapes. With this method, one can trap one single bunch.

In order to accumulate a large number of bunches, positrons have to be slowed down and stored in a dedicated potential well formed by a subset of electrodes of the trap (see Figure 1) before the next bunch arrives. Positrons are cooled by passing through a preloaded electron plasma in another dedicated potential well. This method has been set up and demonstrated in [29] with a continuous positron beam issued

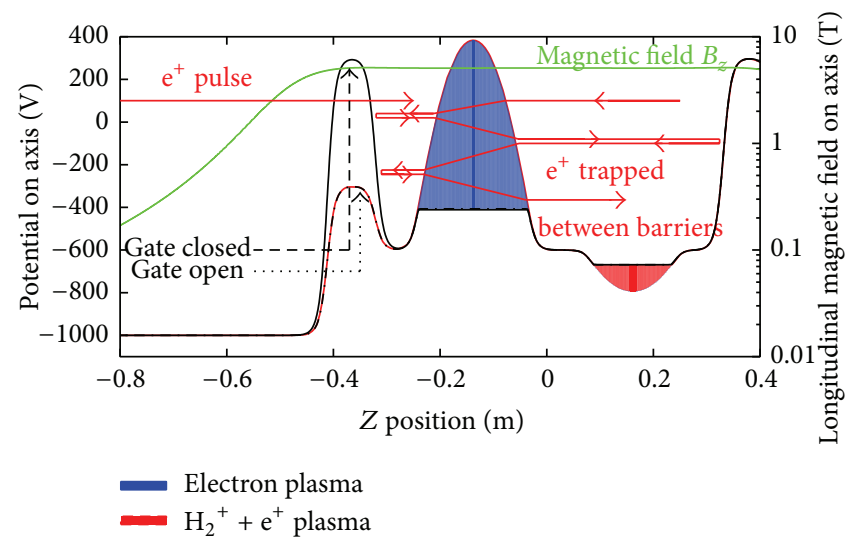

FIGURE 1: The positron trapping mechanism (from [30]). The horizontal axis is the position along the trap axis. The left vertical axis is the voltage seen by particles. The magnetic field strength is shown by the green curve, with the scale on the right vertical axis. In blue, the electron potential well filled with electrons is drawn, reducing the apparent voltage shown by black curve with the value on the left vertical axis. The positron potential is shown well in red. When a positron bunch arrives, the entrance electrode voltage is low (dashed dotted line). It is then increased and positrons go back and forth (it is depicted by red arrows) between this gate and the downstream part of the trap. They pass many times through the electron plasma and are eventually slowed down and fall into their well. The presence of residual $\mathrm{H}_{2}^{+}$helps the final catching of positrons. In order to reach the desired number of trapped positrons, the positron well has to be enlarged and deepened during the accumulation process from around $200 \mathrm{~V}$ to around $1 \mathrm{kV}$. Positrons may also be trapped in the potential well formed between the entrance electrode and the electron well, but its depth remains constant and the induced loss is small.

from a ${ }^{22} \mathrm{Na}$ source. With such a beam, it is not possible to close the entrance gate, and positrons must be slowed down in one step. This was done with a remoderator downstream of the trap. An efficiency of $1 \%$ was obtained. With a bunched positron beam, the remoderator is not necessary, and a much higher efficiency is expected.

The cooling time fixes the maximal LINAC frequency and depends on the density of the electron plasma. With $10^{17} \mathrm{e}^{-} / \mathrm{m}^{3}$, simulations show that the cooling time is around $3 \mathrm{~ms}$.

The principle of this accumulation scheme has now been successfully demonstrated at Saclay. The details of the experimental setup used during accumulation are given in [30]. The result of a successful set of accumulation trials is shown in Figure 2. Given the characteristics of the demonstrator facility at Saclay, a realistic objective is now to accumulate around $10^{8}$ positrons in the trap within 2 minutes.

2.3. Cooling of the $\bar{H}^{+}$Ions. Recent progresses have been made for the design of the cooling of $\overline{\mathrm{H}}^{+}$ions. The cooling proceeds in two steps: Doppler cooling at the $\mathrm{mK}$ level and Raman side band cooling to reach $10 \mu \mathrm{K}$.

In the first step, ions are captured in a linear Paul trap inside which $\mathrm{Be}^{+}$ions are preloaded and laser cooled. In the 


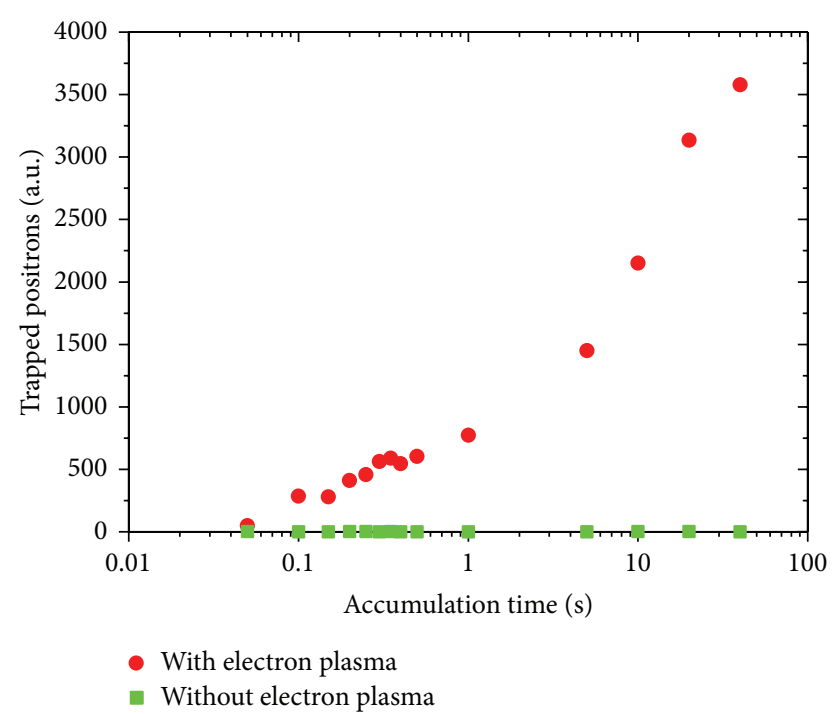

FIGURE 2: The accumulation of positrons (from [30]).

original scheme of GBAR, it was assumed that the $\overline{\mathrm{H}}^{+}$ions would be cooled by Coulomb interactions with the $\mathrm{Be}^{+}$ions (sympathetic cooling). Simulations show that this process is very slow. This is due to the large mass ratio between the ions. As a consequence, one cannot reach the $\mathrm{mK}$ level in a short enough time to avoid the destruction of the $\overline{\mathrm{H}}^{+}$: the laser cooling of $\mathrm{Be}^{+}$induces the photodetachment of the excess positron in a fraction of a second. However, the simulations show also that the addition of a third species of ions of intermediate mass, namely, $\mathrm{HD}^{+}$ions, makes the process efficient enough [31]. Starting with $1800 \mathrm{Be}^{+}$and $200 \mathrm{HD}^{+}$ions, cooling times of $\mathrm{ms}$ are achievable.

In the second step, to reach the $10 \mu \mathrm{K}$ level necessary for the free fall experiment, a $\mathrm{Be}^{+} / \overline{\mathrm{H}}^{+}$ion pair must be transferred to a precision trap to undergo a ground state Raman side band cooling. Calculations show that one may achieve the desired cooling in less than a second. This is shown in [31] and references therein. This method will be tested with matter ions $\left(\mathrm{Ca}^{+} / \mathrm{Be}^{+}, \mathrm{H}_{2}{ }^{+} / \mathrm{Be}^{+}\right)$before being implemented for the GBAR experiment. Traps are being mounted at LKB in Paris and at Mainz University.

Since the uncertainty on the measurement of $\bar{g}$ is fully dominated by the initial velocity dispersion due to both the vertical velocity after cooling and the recoil due to the positron photodetachment, the implementation of a vertical velocity selector will allow a drastic gain in the statistics needed to reach the $1 \%$ precision on $\bar{g}$ as is described in Section 4.

\section{Quantum Reflection of Antihydrogen on Material Surfaces}

In the ultracold regime, the interaction of antihydrogen $(\overline{\mathrm{H}})$ atoms with a surface is governed by the phenomenon of quantum reflection. Although the atoms are strongly attracted to the surface, the atomic wave function can be partly

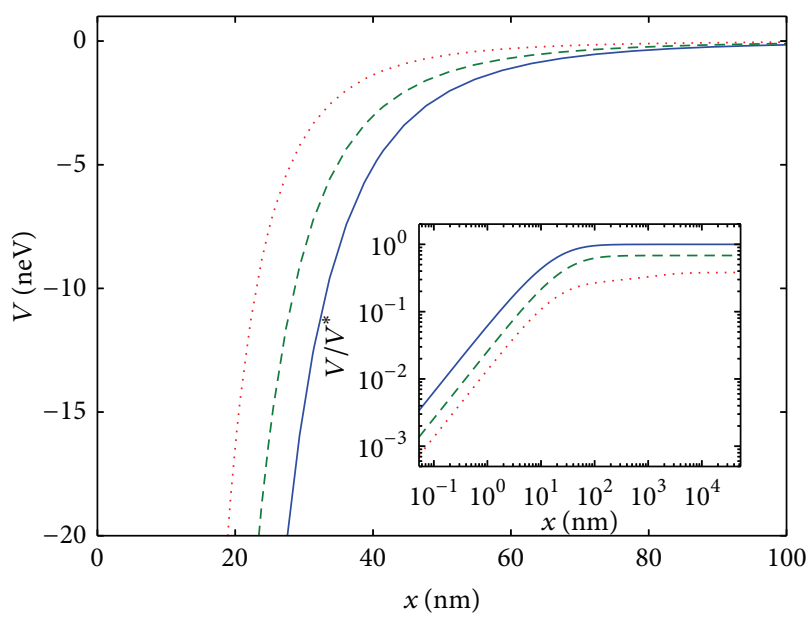

Figure 3: Casimir-Polder (CP) potential for antihydrogen in the vicinity of a material bulk; from top to bottom: perfect conductor (full line), silicon (dashed line), and silica (dotted line); inset: ratio $V / V^{*}$ to the retarded potential $V^{*}$ for a perfectly conducting mirror; see text.

reflected on the steep atom-surface potential, leading to a nonzero probability of classically forbidden reflection. This effect is relevant to experiments such as GBAR where ultracold $\overline{\mathrm{H}}$ atoms are detected by annihilation on a plate (see Section 2).

A single atom placed in vacuum near a material surface experiences an attractive Casimir-Polder (CP) force [32, 33]. This force is a manifestation of the electromagnetic quantum fluctuations which are coupled to the atomic dipole. Quantum reflection occurs if an atom impinges with low velocity on such a rapidly varying potential [34]. We will give a more explicit condition later on.

In this section, we first describe how the CP potential is calculated for realistic experimental conditions. We then go on to compute the scattering amplitudes of an atom on this potential. We show that quantum reflection can be understood as a deviation from the semiclassical approximation. Finally, we describe materials from which quantum reflection is enhanced and above which gravitationally bound states of $\overline{\mathrm{H}}$ could be observed.

3.1. Calculation of the Casimir-Polder Potential. We use the scattering approach to Casimir forces [35] to give a realistic estimation of the atom-surface interaction energy. In this approach, the interacting objects are described by reflection matrices for the electromagnetic field. Reflection on a plane is described by Fresnel coefficients, while reflection on the atom is treated in the dipolar approximation and depends on the dynamic polarizability [36]. This allows an evaluation of the $\mathrm{CP}$ potential for any material when its optical properties are known. Those used here are detailed in [37]. Note that since the typical length scale for quantum reflection $(\sim 100 \mathrm{~nm})$ is below the thermal wavelength at $300 \mathrm{~K}(\sim 1 \mu \mathrm{m})$, we carried out all calculations at null temperature.

The CP potential for $\overline{\mathrm{H}}$ at a distance $x$ of a perfectly conducting plane and thick silicon and silica slabs are presented in Figure 3. For a perfectly conducting mirror in 
the long-distance regime, we recover the historic result of Casimir and Polder [32, 33]:

$$
V(x) \underset{x \gg \lambda}{\approx} V^{*}(x)=-\frac{3 \hbar c}{8 \pi x^{4}} \frac{\alpha(0)}{4 \pi \epsilon_{0}},
$$

where $\alpha(0)$ is the static polarizability of the atom.

For real mirrors, the potential is reduced but it shows the same power law dependence in the van der Waals (short distance) and retarded (long-distance) regimes:

$$
V(x) \underset{x \ll \lambda}{\approx}-\frac{C_{3}}{x^{3}}, \quad V(x) \underset{x \gg \lambda}{\approx}-\frac{C_{4}}{x^{4}},
$$

where $\lambda$ is a typical wavelength associated with the optical response of atom and plane.

3.2. Scattering on the Casimir-Polder Potential. We now solve the Schrödinger equation for an atom of energy $E>0$ scattering on the CP potential $V(x)$ :

$$
\frac{\mathrm{d}^{2}}{\mathrm{~d} x^{2}} \psi(x)+\frac{p(x)^{2}}{\hbar^{2}} \psi(x)=0
$$

with $p(x)=\sqrt{2 m(E-V(x))}$ being the classical momentum. We write the exact wave function as a sum of counterpropagating $\mathrm{WKB}$ waves whose coefficients are allowed to vary:

$$
\begin{aligned}
\psi(x)= & \frac{c_{\text {in }}(x)}{\sqrt{p(x)}} \exp \left(-\frac{i}{\hbar} \int^{x} p\left(x^{\prime}\right) \mathrm{d} x^{\prime}\right) \\
& +\frac{c_{\text {out }}(x)}{\sqrt{p(x)}} \exp \left(\frac{i}{\hbar} \int^{x} p\left(x^{\prime}\right) \mathrm{d} x^{\prime}\right) .
\end{aligned}
$$

Upon insertion in the Schrödinger equation, we obtain coupled first-order equations for the coefficients $c_{\text {in }}(x), c_{\text {out }}(x)$ [38]. The annihilation of $\overline{\mathrm{H}}$ on the material surface translates as a fully absorbing boundary condition on the surface: $c_{\text {out }}(x=0)=0$. This is in contrast with matter atoms, for which more complicated surface physics is involved in the boundary condition. We emphasize that $\overline{\mathrm{H}}$-surface interaction at distances below few atomic units differs significantly from $H$-surface interaction. It is remarkable that, due to complete annihilation of $\overline{\mathrm{H}}$ in the bulk of the surface, the details of such short-range physics are not important [39]. Indeed, any information about antiatom-wall interaction is encrypted in the reflected wave, which, as we will show in the following, is produced at asymptotically large distances.

Close to the surface, the energy becomes negligible compared with the potential, which takes the van der Waals form and $c_{\text {in }}(x), c_{\text {out }}(x)$ can be solved analytically [37]. The equations are then integrated numerically until $c_{\text {in }}(x), c_{\text {out }}(x)$ become constants.

The reflection probability $|r|^{2}=\lim _{x \rightarrow \infty}\left|c_{\text {out }}(x) / c_{\text {in }}(x)\right|^{2}$ is plotted against the energy $E$ in Figure 4 for various semiinfinite media. Note that the quantum reflection probability is larger for materials with a weaker CP interaction, such as silica.

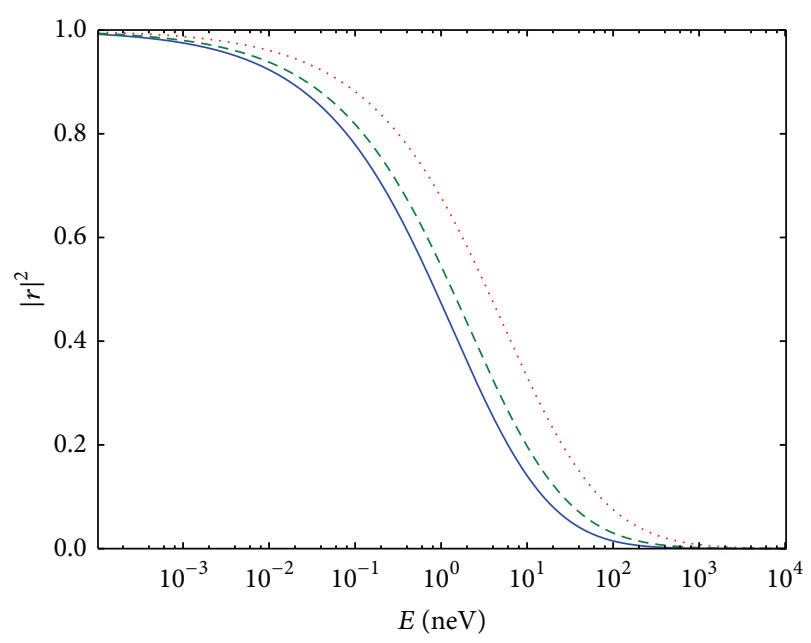

FIGURE 4: Quantum reflection probability $|r|^{2}$ as a function of the energy for antihydrogen atoms on bulk mirrors; from bottom to top: perfect conductor (full line), silicon (dashed line), and silica (dotted line).

3.3. The Badlands Function. To understand this surprising result, we look more closely at what distinguishes the exact solution of the Schrödinger equation from the reflectionless $\mathrm{WKB}$ approximation. If $c_{\text {in }}, c_{\text {out }}$ are no longer allowed to vary, one can show that the wave function (7) obeys a modified Schrödinger equation where $p(x)^{2}$ is replaced by $\widetilde{p}(x)^{2}=$ $p(x)^{2}(1+Q(x))$ [38]. $Q(x)$ is known as the badlands function since the $\mathrm{WKB}$ approximation is not valid in regions where it is nonnegligible:

$$
Q(x)=\frac{\hbar^{2}}{2 p(x)^{2}}\left(\frac{p^{\prime \prime}(x)}{p(x)}-\frac{3}{2} \frac{p^{\prime}(x)^{2}}{p(x)^{2}}\right) .
$$

For the CP potential, the badlands function exhibits a peak in the region where $|V(x)|=E$ but goes to zero both as $x \rightarrow \infty$ (where the potential cancels) and as $x \rightarrow 0$ (where the classical momentum diverges).

As the energy is decreased, the semiclassical approximation breaks down and the badlands function's peak becomes larger. But, for a given energy, the peak is larger and closer to the surface when the potential is weak, as shown in Figure 5. The difference between exact and WKB solutions is larger in weaker CP potentials, leading to enhanced quantum reflection.

3.4. Enhancing Quantum Reflection. Quantum reflection first appears as a bias in the context of the GBAR experiment, since it tends to exclude low energy atoms from the statistics. However, this phenomenon opens perspectives for the storage and guiding of antimatter with material walls. With this in mind, we consider materials which couple weakly to the electromagnetic field and are therefore good mirrors for atoms, as we have seen in the previous paragraph.

A simple strategy is to remove matter from the reflective medium, by using thin slabs or porous materials for example. Our versatile approach allowed us to compute the $\mathrm{CP}$ 


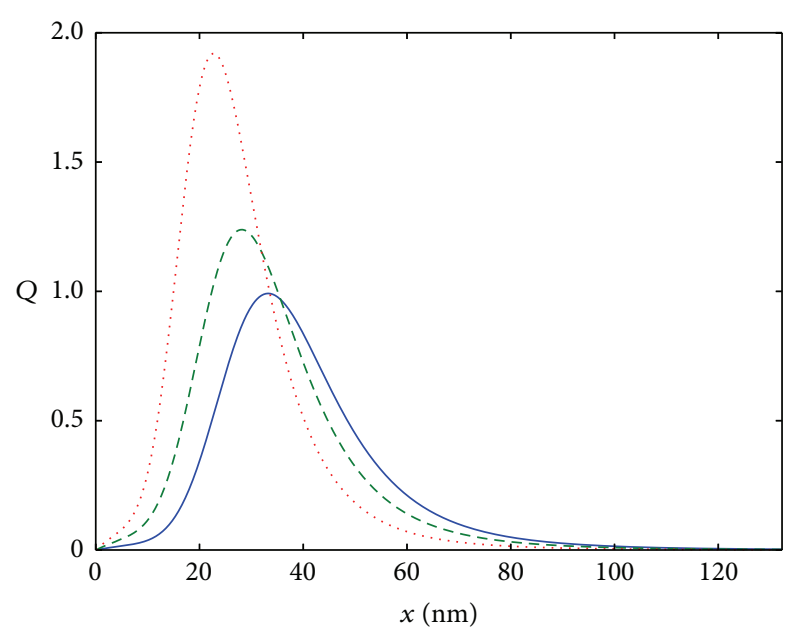

FIGURE 5: The badlands function $Q(x)$ for an antihydrogen atom with energy $E=10 \mathrm{neV}$; from bottom to top: perfect conductor (full line), silicon (dashed line), and silica (dotted line).

TABLE 1: The lifetime of the first gravitationally bound state of antihydrogen above various surfaces.

\begin{tabular}{lc}
\hline Surface (porosity) & Lifetime (s) \\
\hline Perfect conductor & 0.11 \\
Bulk silicon & 0.14 \\
Bulk silica & 0.22 \\
Nanodiamond powder (95\%) & 0.89 \\
Porous silicon (95\%) & 0.94 \\
Silica aerogel (98\%) & 4.6 \\
\hline
\end{tabular}

interaction near thin slabs, an undoped graphene sheet [37] and nanoporous materials [40]. The latter consist of a solid matrix which forms an array of nanometric pores. Aerogels, which are obtained by supercritically drying a silica gel, are a well-known example. We also consider porous silicon and powders of diamond nanoparticles formed by explosive shock.

From a distance larger than the typical pore size, such materials can be modeled as homogeneous effective media with properties averaged between that of vacuum and that of the solid matrix. In consequence, their effective dielectric constant is extremely low, as a result of which quantum reflection is exceptionally efficient. In Table 1 , we show the lifetime of an antihydrogen atom in the first gravitationally bound state above a surface (see Section 5 for more details).

Note that this approach does not take into account the possible presence of stray charges on the surface, a question that would have to be addressed to observe the predicted reflection probabilities. Moreover, the effective medium approximation is applicable only for low atom velocities, such that the atom is reflected far enough from the surface. With these caveats, nanoporous materials are an outstanding candidate for the manipulation and study of antihydrogen and its gravitationally bound states over lifetimes of a few seconds.

\section{Shaping of Vertical Velocity Components of Antihydrogen Atoms for GBAR}

The main source of uncertainty on the determination of $\bar{g}$ in the GBAR experiment is the width of the vertical velocity distribution of the atom at the beginning of the free fall. This spread in velocities is due to the quantum uncertainty on the momentum of $\overline{\mathrm{H}}^{+}$in the ground state of the harmonic Paul trap and to the additional recoil associated with the photodetachment of the extra positron (see Section 2).

In this section, we give an estimation of the uncertainty on the arrival time associated with the initial vertical velocity spread and show how it can be reduced by filtering out the fastest atoms. Since slow antihydrogen atoms bounce on material surfaces thanks to quantum reflection (see previous section), the filtering scheme used in GRANIT with ultracold neutrons [21] can also be applied in GBAR.

4.1. Width of the Arrival Time Distribution. We consider a wave packet falling in a linear gravitational potential and want to determine the arrival time distribution on a fixed horizontal plane, supposing there is no reflection from that (ideal) detector. In this case, classical and quantum calculations give identical results, as can be seen by noticing that the Wigner quasidistribution function obeys the classical equations of motion if the potential is at most quadratic. Therefore, in a linear potential, a given initial phase-space distribution simply propagates along the classical trajectories.

For a wave packet initially centered at a height $H$ above the detector, with zero mean velocity and uncorrelated vertical position and velocity distributions of widths $\Delta z$ and $\Delta v$, respectively, the spread of the arrival time distribution is

$$
\frac{\Delta t}{t_{H}}=\sqrt{\left(\frac{\Delta z}{2 H}\right)^{2}+\left(\frac{\Delta v}{\sqrt{2 \bar{g} H}}\right)^{2}},
$$

with $t_{H}=\sqrt{2 H / \bar{g}}$ being the classical free fall time. This translates as a statistical uncertainty $\Delta \bar{g} / \bar{g}=2 \Delta t / \sqrt{N} t_{H}$ on the determination of $\bar{g}$ after $N$ independent measurements.

If the particle is initially in the ground state of a harmonic trap, the distribution is Gaussian and saturates the Heisenberg inequality: $\Delta z \Delta v=\hbar / 2 m$. Then, the time uncertainty is minimal for

$$
\Delta v=\Delta v_{\mathrm{opt}}=\sqrt{\frac{\hbar}{2 m} \sqrt{\frac{\bar{g}}{2 H}}} .
$$

For $H=30 \mathrm{~cm}$ and $\bar{g}=g$, this evaluates $\Delta v_{\mathrm{opt}} \approx 3.6 \times$ $10^{-4} \mathrm{~m} / \mathrm{s}$, and the relative uncertainty on the arrival time is $2 \times 10^{-4}$. However, the current expected value for GBAR is three orders of magnitude larger $\Delta v_{0} \approx 0.5 \mathrm{~m} / \mathrm{s}$, which leads to a relative uncertainty of 0.2 .

The uncertainty in GBAR is largely dominated by the vertical velocity dispersion. If the initial velocity dispersion can be reduced from $\Delta v_{0}$ to $\Delta v$ by filtering out the hottest atoms, the single-shot precision and the number of atoms are both reduced by a factor $\Delta v / \Delta v_{0}$. Despite the loss in statistics, 


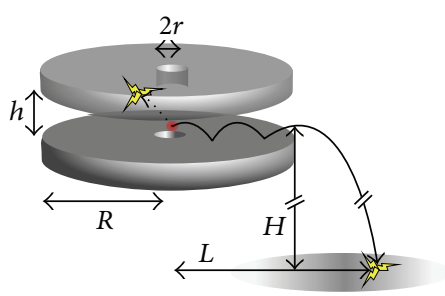

FIgURE 6: Scheme of the proposed device to reduce the vertical velocity spread of the falling wave packet (see text).

this results in a net reduction of the statistical uncertainty on $\bar{g}$.

4.2. Shaping of the Vertical Velocity Distribution. Our proposal [41] to realize this filtering is to let the atoms pass through a horizontal slit between two disks. The bottom disk has a smooth top surface on which atoms reflect with high probability whereas the top disk has a rough bottom surface which effectively acts as an absorber for the atoms (see Figure 6). Antihydrogen is initially trapped in the center of the two disks (openings are made in the center of the disks to allow operation of the trap). If its vertical velocity is high enough to reach the rough surface, it is reflected nonspecularly and remains inside the device until it annihilates with high probability. On the contrary, if it cannot reach the top disk, the atom will exit the device with high probability after bouncing on the bottom mirror a few times. It then falls freely to a detector located at a height $H$ below. Since the horizontal velocity is conserved, the knowledge of the total time between photodetachment and annihilation and of the total horizontal $L$ distance traveled allows one to correct for the time spent inside the device before the free fall.

If $h$ is the height of the slit, the velocity spread at the output is $\Delta v \approx \sqrt{2 \bar{g} h}$ and the proportion of atoms that exit the device is $N / N_{0} \approx \Delta v / \sqrt{2 \pi} \Delta v_{0}$. Using the shaping device therefore reduces the statistical uncertainty on $\bar{g}$ by a factor which scales $h^{1 / 4}$.

Classically, going to ever smaller slit heights leads to arbitrarily good precision. For example, for $h=1 \mathrm{~mm}, \Delta v \approx$ $0.14 \mathrm{~m} / \mathrm{s}, N / N_{0} \approx 5 \%$, and the accuracy is improved by a factor 2, whereas, for $h=50 \mu \mathrm{m}, \Delta v \approx 0.03 \mathrm{~m} / \mathrm{s}, N / N_{0} \approx 1 \%$, and the accuracy improved by a factor 4 .

There are, however, two limits: the number of repetitions of the experiment must be large enough that at least some atoms make it through the filter, but, more fundamentally, the wave function of the atom must fit inside the slit. Indeed for slit sizes below $50 \mu \mathrm{m}$ the discrete spectrum of states bound by gravity must be taken into account. For a slit size of $20 \mu \mathrm{m}$, only the ground state can travel through the guide; below that, the transmission drops to zero.

This fact has been used to demonstrate the existence of gravitationally bound states for neutrons [21]. The next section explores the possibilities of similar experiments with antihydrogen to further increase the precision of equivalence principle tests on antimatter.

\section{Resonance Spectroscopy of Gravitational States of Antihydrogen Near Material Surface}

In this section, we will study a motion of an $\overline{\mathrm{H}}$ atom, localized in a gravitational state near a horizontal plane mirror. The existence of such states though counterintuitive is explained by the phenomenon of quantum reflection of ultracold (anti-)atoms from a steep attractive Casimir-Polder atomsurface potential. Such states have similar properties with those discovered for neutrons [21, 42-45].

To account for the interaction of $\overline{\mathrm{H}}$ with a material wall, the gravitational quantum states (1) receive a complex energy shift $\varepsilon_{0} \Delta$, with $\Delta \simeq-i 0.005$ for a perfectly conducting wall [46]. All states, therefore, acquire equal shift and width, which is a function of a material surface substance $\Gamma=2|\Delta| \varepsilon_{0}$. This width corresponds to the lifetime of $0.1 \mathrm{~s}$ in case of a perfectly conducting surface and is twice longer for silica [37, $39,40]$, for instance. The equal shifts of all gravitational states energy levels mean that already small modification of gravitational states due to antiatom-surface interaction is canceled out in the transition frequencies. This makes resonance spectroscopy of gravitational states particularly interesting for measuring gravitational properties of $\overline{\mathrm{H}}$.

The interest to study gravitational quasistationary states of $\overline{\mathrm{H}}$ is due to their comparatively long life-time on one hand and easy identification of certain state because of it is mesoscopic spatial scale. This opens an interesting perspective to apply potentially very precise resonance spectroscopy method to establish the gravitational properties of antiatoms. These methods are based on inducing an observation of resonance transitions between gravitational states. One possible approach is to use an alternating inhomogeneous magnetic field for such a purpose.

The interaction of a magnetic field with a ground state $H$ atom moving through the field [47-49] is dominated by the interaction of an average magnetic moment of the atom [20] in a given hyperfine state with the magnetic field. We are going to focus on an alternating magnetic field with a gradient in the vertical direction. This condition is needed for coupling the field and the center of mass (c.m.) $\overline{\mathrm{H}}$ motion in the gravitational field of the Earth. It allows one to induce resonant transitions between quantum gravitational states of $\overline{\mathrm{H}}[46]$.

We will consider the magnetic field in the following form:

$$
\vec{B}(z, x, t)=B_{0} \vec{e}_{z}+\beta \cos (\omega t)\left(z \vec{e}_{z}-x \vec{e}_{x}\right) .
$$

Here, $B_{0}$ is the amplitude of a constant, vertically aligned, component of magnetic field, $\beta$ is the value of magnetic field gradient, $z$ is a distance measured in the vertical direction, and $x$ is a distance measured in the horizontal direction, parallel to the surface of a mirror. A time-varying magnetic field (11) is accompanied with an electric field $([\vec{\nabla} \vec{E}]=$ $-(1 / c) \partial \vec{B} / \partial t)$. However, for the velocities of ultracold atoms, corresponding interaction terms are small and thus will be omitted.

An inhomogeneous magnetic field couples the spin and the spatial degrees of freedom. A $\overline{\mathrm{H}}$ wave function is 
described in this case using a four-component column (in a nonrelativistic treatise) in the spin space, each component being a function of the c.m. coordinate $\vec{R}$, relative $\bar{p}-\bar{e}$ coordinate $\vec{\rho}$, and time $t$. The corresponding Schrödinger equation is

$$
\begin{aligned}
& i \hbar \frac{\partial \Phi_{\alpha}(\vec{R}, \vec{\rho}, t)}{\partial t} \\
& =\sum_{\alpha^{\prime}}\left[-\frac{\hbar^{2}}{2 m} \Delta_{R}+M g z\right. \\
& \left.\quad+V_{\mathrm{CP}}(z)+\widehat{H}_{\mathrm{in}}+\widehat{H}_{m}\right]_{\alpha, \alpha^{\prime}} \Phi_{\alpha^{\prime}}(\vec{R}, \vec{\rho}, t) .
\end{aligned}
$$

A subscript $\alpha$ in this equation indicates one of four spin states of the $\bar{p}-\bar{e}$ system. The meaning of the interaction terms is the following. $V_{\mathrm{CP}}(z)$ is an atom-mirror interaction potential, which turns into the Casimir-Polder potential at an asymptotic atom-mirror distance (see $[39,50]$ and references therein). $\widehat{H}_{\text {in }}$ is the Hamiltonian of the internal motion, which includes the hyperfine interaction:

$$
\widehat{H}_{\text {in }}=-\frac{\hbar^{2}}{2 \mu} \Delta_{\rho}-\frac{e^{2}}{\rho}+\frac{\alpha_{\mathrm{HF}}}{2}\left(\widehat{F}^{2}-\frac{3}{2}\right) .
$$

Here, $\mu=m_{1} m_{2} / m, m_{1}$ is the antiproton mass, $m_{2}$ is the positron mass, $m=m_{1}+m_{2}, \alpha_{\mathrm{HF}}$ is the hyperfine constant, and $\widehat{F}$ is the operator of the total spin of the antiproton and the positron. We will treat only $\overline{\mathrm{H}}$ atoms in a $1 S$-state (below, we will show that the excitation of other states in the studied process is improbable). The term $\left(\alpha_{\mathrm{HF}} / 2\right)\left(\widehat{F}^{2}-\right.$ $3 / 2$ ) is a model operator, which effectively accounts for the hyperfine interaction and reproduces the hyperfine energy splitting correctly. The term $\widehat{H}_{m}$ describes the field-magnetic moment interaction:

$$
\widehat{H}_{m}=-2 \vec{B}(z, x, t)\left(\mu_{\bar{e}} \widehat{s}_{\bar{e}} \times \widehat{I}_{\bar{p}}+\mu_{\bar{p}} \widehat{s}_{\bar{p}} \times \widehat{I}_{\bar{e}}\right) .
$$

Here, $\mu_{\bar{e}}$ and $\mu_{\bar{p}}$ are magnetic moments of the positron and the antiproton, respectively, $\widehat{s}_{\bar{e}}, \widehat{s}_{\bar{p}}$ is a spin operator, acting on spin variables of positron (antiproton), and $\widehat{I}_{\bar{e}}, \widehat{I}_{\bar{p}}$ is a corresponding identity operator. As far as the field $\vec{B}(z, x, t)$ changes in space and in time, this term couples the spin and the c.m. motion.

We will assume that in typical conditions of a spectroscopy experiment the $\overline{\mathrm{H}}$ velocity component $v$ parallel to the mirror surface (directed along $x$-axis) is of the order of a few $\mathrm{m} / \mathrm{s}$ and is much larger than a typical vertical velocity in lowest gravitational states (which is of the order of $\mathrm{cm} / \mathrm{s}$ ). We will treat the motion in a frame moving with the velocity $v$ of the $\overline{\mathrm{H}}$ atom along the mirror surface. Thus, we are going to consider the $x$-component motion as a classical motion with a given velocity $v$, and we will substitute a $x$-dependence by a $t$-dependence. We will also assume that $B_{0} \gg \beta L$, where $L \sim 30 \mathrm{~cm}$ is a typical size of an experimental installation of interest. This condition is needed for "freezing" the magnetic moment of an atom along the vertical direction; it provides the maximum transition probability.
We will be interested in the weak field case, such that the Zeeman splitting is much smaller than the hyperfine level spacing $\mu_{B} B_{0} \ll \alpha_{\mathrm{HF}}$. The hierarchy of all mentioned above interaction terms could be formulated as follows:

$$
\frac{m_{2} e^{2}}{\hbar^{2}} \gg \alpha_{\mathrm{HF}} \gg \mu_{\bar{e}}\left|B_{0}\right| \gg E_{n}
$$

and, thus, it justifies the use of the adiabatic expansion for solving (12); it is based on the fact that an internal state of an $\overline{\mathrm{H}}$ atom follows adiabatically the spatial and temporal variations of an external magnetic field. Neglecting nonadiabatic couplings, an equation system for the amplitude $C_{n}(t)$ of a gravitational state $\psi_{n}(z)$ has the following form:

$$
i \hbar \frac{d C_{n}(t)}{d t}=\sum_{k} C_{k}(t) V_{n, k}(t) \exp \left(-i \omega_{n k} t\right) .
$$

The transition frequency $\omega_{n k}=\left(E_{k}-E_{n}\right) / \hbar$ is determined by the gravitational energy level spacing. This fact is used in the proposed approach to access the gravitational level spacing by means of scanning the applied field frequency, as will be explained in the following.

Within this formalism, the role of the coupling potential $V(z, t)$ is played by the energy of an atom in a fixed hyperfine state thought of as a function of (slowly varying) distance $z$ and time $t$. Consider

$$
V_{n, k}(t)=\int_{0}^{\infty} \psi_{n}(z) \psi_{k}(z) E(t, z) \mathrm{d} z .
$$

Here, $\psi_{n}(z)$ is the gravitational state wave function, which is known in terms of the Airy function [46].

The energy $E(z, t)$ is the eigenvalue of the internal and magnetic interactions $\widehat{H}_{\text {in }}+\widehat{H}_{m}$, where the c.m. coordinate $\vec{R}$ and time $t$ are treated as slow-changing parameters. Corresponding expressions for the eigenenergies of a $1 S$ manifold are

$$
\begin{aligned}
& E_{a, c}=E_{1 s}-\frac{\alpha_{\mathrm{HF}}}{4} \mp \frac{1}{2} \sqrt{\alpha_{\mathrm{HF}}^{2}+\left|\left(\mu_{B}-\mu_{\bar{p}}\right) B(z, t)\right|^{2}}, \\
& E_{b, d}=E_{1 s}+\frac{\alpha_{\mathrm{HF}}}{4} \mp \frac{1}{2}\left|\left(\mu_{B}+\mu_{\bar{p}}\right) B(z, t)\right| .
\end{aligned}
$$

Subscripts $a, b, c, d$ are standard notations for hyperfine states of a $1 S$ manifold in a magnetic field. The presence of a constant field $B_{0}$ produces the Zeeman splitting between states $b$ and $d$. As far as the energy of states $b, d$ depends on magnetic field linearly, while, for states $a, c$, it depends on magnetic field quadratically, only transition between $b, d$ states takes place in case of a weak field. In the following, we will consider only transitions between gravitational states in a $1 S(b, d)$ manifold.

A qualitative behavior of the transition probability is given in the Rabi formula, which can be deduced by means of neglecting the high frequency terms compared to the resonance couplings of only two states, initial $i$ and final $f$, 


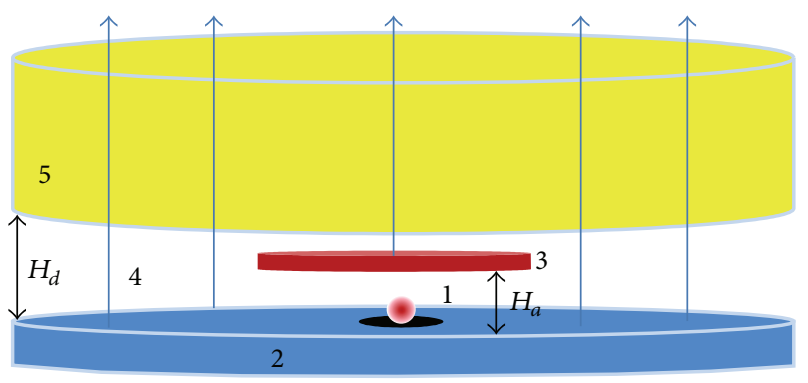

FIGURE 7: A sketch of the principle scheme of an experiment on magnetically induced resonant transitions between $\overline{\mathrm{H}}$ gravitational states. 1: a source of ultracold antihydrogen, 2: a mirror, 3: an absorber, 4: a magnetic field, and 5: a detector.

in case the field frequency $\omega$ is close to the transition frequency $\omega_{i f}=\left(E_{f}-E_{i}\right) / \hbar$ :

$$
\begin{aligned}
P= & \frac{1}{2} \frac{\left(V_{i f}\right)^{2}}{\left(V_{i f}\right)^{2}+\hbar^{2}\left(\omega-\omega_{i f}\right)^{2}} \\
& \times \sin ^{2}\left(\frac{\sqrt{\left(V_{i f}\right)^{2}+\hbar^{2}\left(\omega-\omega_{i f}\right)^{2}}}{2 \hbar} t\right) \exp (-\Gamma t) .
\end{aligned}
$$

The factor $1 / 2$ appears in front of the right-hand side of the above expression due to the fact that only two $(b, d)$ of four hyperfine states participate in the magnetically induced transitions.

It is important that the transition frequencies $\omega_{i f}$ do not depend on the antiatom-surface interaction up to the second order in the splitting $\Delta$. This is a consequence of the already mentioned fact that all energies of gravitational states acquire equal shift due to the interaction with a material surface.

A resonant spectroscopy of $\overline{\mathrm{H}}$ gravitational states could consist of observing $\overline{\mathrm{H}}$ atoms localized in the gravitational field above a material surface at a certain height as a function of the applied magnetic field frequency. A "flow-through type" experiment, analogous to the one discussed for the spectroscopy of neutron gravitational states [51], includes three main steps. A sketch of a principle scheme of an experiment proposed in [41] is shown in Figure 7 (see also Section 4).

First, an atom of $\overline{\mathrm{H}}$ is shaped in a ground gravitational state. This is achieved by means of passing $\overline{\mathrm{H}}$ through a slit, formed by a mirror and an absorber, which is placed above the mirror at a given height $H_{a}$. The mirror and the absorber form a waveguide with a state-dependent transmission [45]. The choice of $H_{a}=H_{1} \simeq 13.6 \mu \mathrm{m}$ implies that only $\overline{\mathrm{H}}$ atoms in the ground gravitational state pass through the slit. Second, $\overline{\mathrm{H}}$ atoms are affected by an alternating magnetic field (11) while they are moving parallel to the mirror. An excited gravitational state is resonantly populated. Third, the number of $\overline{\mathrm{H}}$ atoms in an excited state is measured by means of counting the annihilation events in a detector, which is placed at a height $H_{d}$ above the mirror. The value of $H_{d}$ is chosen

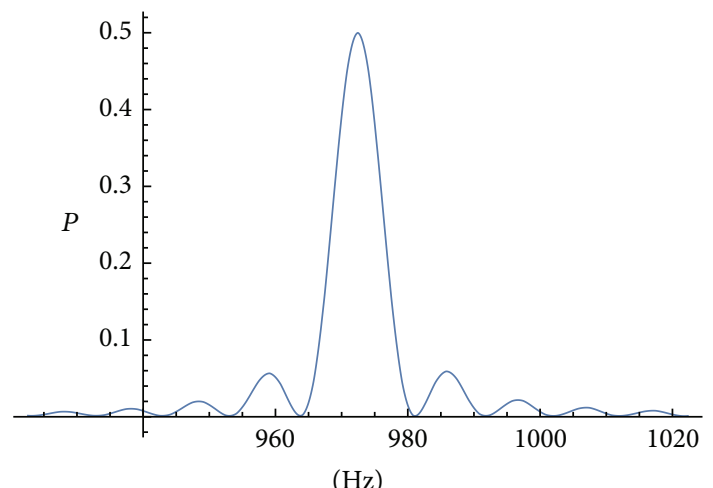

FIGURE 8: The transition probability as a function of the magnetic field frequency for the transition from the ground state to 6 th gravitational state.

to be larger than the spatial size of the gravitational ground state and smaller than the spatial size of the final state (2), $H_{1} \ll H_{d}<H_{f}$, so that the ground state atoms pass through, while atoms in the excited state are detected.

We present a simulation of the number of detected annihilation events as a function of the field frequency in Figure 8 for the transition from the ground to the 6 th excited state, based on a numerical solution of the equation system (16). The corresponding resonance transition frequency is $\omega=972.46 \mathrm{~Hz}$. The value of the field gradient, optimized to obtain the maximum probability of $1 \rightarrow 6$ transition during the time of flight $t_{\mathrm{fl}}=\tau=0.1 \mathrm{~s}$ turned to be equal to $\beta=$ $27.2 \mathrm{Gs} / \mathrm{m}$; the corresponding guiding field value, which guarantees the adiabaticity of the magnetic moment motion, is $B_{0}=30 \mathrm{Gs}$.

It follows from (1) that the $\overline{\mathrm{H}}$ gravitational mass could be deduced from the measured transition frequency $\omega_{n k}$ as follows:

$$
M=\sqrt{\frac{2 m \hbar \omega_{n k}^{3}}{g^{2}\left(\lambda_{k}-\lambda_{n}\right)^{3}}} .
$$

Let us mention that $g$ in the above formula means the gravitational field intensity near the Earth's surface, a value which characterizes properties of the field and is assumed to be known with a high precision. At the same time, all the information about gravitational properties of $\overline{\mathrm{H}}$ is included in the gravitational mass $M$. Equality of the gravitational mass $M$ and the inertial mass $m$, imposed by the equivalence principle, results in the following expression:

$$
M=\frac{2 \hbar \omega_{n k}^{3}}{g^{2}\left(\lambda_{k}-\lambda_{n}\right)^{3}} .
$$

Estimation of the accuracy of the above expression requires accounting for different effects, including dynamical Stark shift of the resonance line, nonadiabatic corrections to the transition probability, and interaction of alternating magnetic field with a mirror. The detailed study of different systematic effects is under way. Assuming that the spectral 


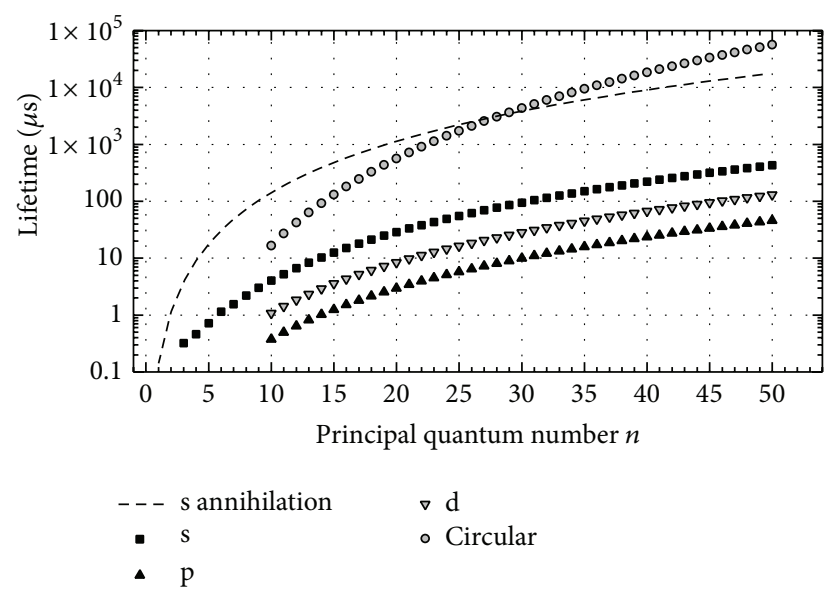

FIGURE 9: Radiative lifetimes of various Ps states as a function of the principal quantum number $n$. The lifetimes were calculated by summing the Einstein $A$ coefficients of all electric-dipole-allowed decay channels from each Rydberg state. For each $A$ coefficient the appropriate radial integrals were determined using analytic expressions for the radial wave functions in a pure Coulomb potential [53]. The dashed line is the annihilation lifetime of $n \mathrm{~s}$ states. After [5].

line width is determined by the lifetime $\tau \approx 0.1 \mathrm{~s}$ of gravitational states, we estimate that the gravitational mass $M$ can be deduced with the relative accuracy $\epsilon_{M} \sim 10^{-3}$ for 100 annihilation events for the transition to the 6 th state.

\section{Gravitational Free Fall of Cold Positronium}

Antihydrogen, muonium, and positronium are the possible candidates for gravity measurements on antimatter, with various pros and cons. Antihydrogen and muonium [4] are extremely difficult to produce, requiring large facilities (i.e., PSI, CERN), whereas positronium is relatively easy to produce in smaller university laboratories. However, Ps has an inconvenient propensity to self-annihilate; the triplet ground state vacuum lifetime of only 142 ns would seem to preclude using this system for a free fall measurement. As has been pointed out by various authors, in particular Mills, Jr., [52], this is not the case, since one need only excite Ps atoms into long-lived Rydberg states to prevent self-annihilation. Indeed, for any Ps state with $n>1$ the radiative lifetime is always less than the annihilation lifetime. The only excited state for which this is not true is the metastable $2 \mathrm{~s}$ state. That is to say, for excited states, the overlap of the positron and electron wave functions is sufficiently low that annihilation can be considered to be negligible (see Figure 9).

The radiative lifetimes of excited Ps states, shown in Figure 9, are almost twice those of the corresponding states in hydrogen. For practical reasons, the smallest Ps beam deflections one can expect to observe will be 10 s of micrometers or more. Therefore, if Ps falls with the usual gravitational acceleration, it would be necessary to produce states with lifetimes of the order of a few ms to observe such deflections. As is evident from Figure 9, achieving such long radiative
TABLE 2: The $n$-dependence of several properties of Rydberg atoms, with examples shown for the $30 \mathrm{~d}$ state of Ps, $\mathrm{H}$, and He. The state separation is calculated for $30 \mathrm{~d} \rightarrow 31 \mathrm{~d}$. The orbital radius is defined here as the expectation value $\langle r\rangle=(1 / 2)\left(3 n_{\mathrm{eff}}^{2}-l(l+1)\right)$, where $n_{\mathrm{eff}}$ includes the relevant quantum defect. The electric dipole momentto-mass ratios are calculated for the outermost state of the $n=30$, $m=2$ Stark manifold. The radiative lifetime $n$-dependence applies only to low $l$ states: for circular states, the scaling is closer to $n^{5}$ (see Figure 9).

\begin{tabular}{lcccc}
\hline & $n$-Scaling & Ps & H & He \\
\hline Binding energy $(\mathrm{meV})$ & $n^{-2}$ & -7.56 & -15.11 & -15.12 \\
\hline State separation $(\mathrm{meV})$ & $n^{-3}$ & 0.48 & 0.96 & 0.96 \\
\hline Orbital radius $\left(a_{0}\right)$ & $n^{2}$ & 2694 & 1347 & 1347 \\
\hline Radiative lifetime $(\mu \mathrm{s})$ & $n^{3}$ & 28.4 & 14.2 & 12.2 \\
\hline Dipole moment $/ \mathrm{mass}\left(e a_{0} / \mathrm{amu}\right)$ & $n^{2}$ & $2.2 \times 10^{6}$ & 1206 & 304 \\
\hline
\end{tabular}

lifetimes requires exciting either low $l$ Rydberg levels (i.e., $s$ or d) to extremely high principal quantum numbers, or going to lower $n$ states (perhaps around $n=30$ or so) and then transferring the atoms to circular states, or if not true circular states, at least states with higher angular momentum. For a discussion of the properties of circular states and methods for producing them, see [54].

Aside from the creation of sufficiently long-lived Rydberg levels, conducting a Ps free fall experiment will require solving many other problems. In order to accomplish an experiment of the type first outlined by Mills and Leventhal [55], it will be necessary to produce a small (10-50 micron) "point" source of slow positronium in a cryogenic environment. The resulting long-lived Rydberg atoms will then have to be formed into a beam, perhaps by electrostatic manipulation (focusing and deceleration) via their electric dipole moments [56], and finally detected with good spatial resolution (as a function of flight time) in order to observe a deflection due to gravity. A schematic view of such an experiment is presented in Figure 10. Possible methods to accomplish some of these tasks are considered elsewhere $[5,55]$.

The production of Ps Rydberg states with principal quantum numbers around 30 can be accomplished using a two-step process ( $1 \mathrm{~s} \rightarrow 2 \mathrm{p} \rightarrow n \mathrm{~d})$ and has already been experimentally demonstrated using broadband $(\sim 100 \mathrm{GHz})$ lasers to accommodate the large Doppler-broadened width of the transitions [57]. However, this methodology is not well suited to the requirement that these atoms are subsequently transferred to higher angular momentum states, and, in order to achieve the required state selectivity, it may be necessary to use a different excitation mechanism; that is, a Dopplerfree two-photon transition from the ground state directly to a well-defined Rydberg Stark state [58].

As is well known, Rydberg atoms exhibit exaggerated properties [54] (see Table 2). In the present case, this is critical, since we seek to produce Ps atoms with very long lifetimes, and also take advantage of the large electric dipole moments of Rydberg atoms to create and control an atomic beam. However, insofar as we are compelled to make use of electrically neutral systems to measure the weak gravitational force acting on antimatter particles without extraneous 


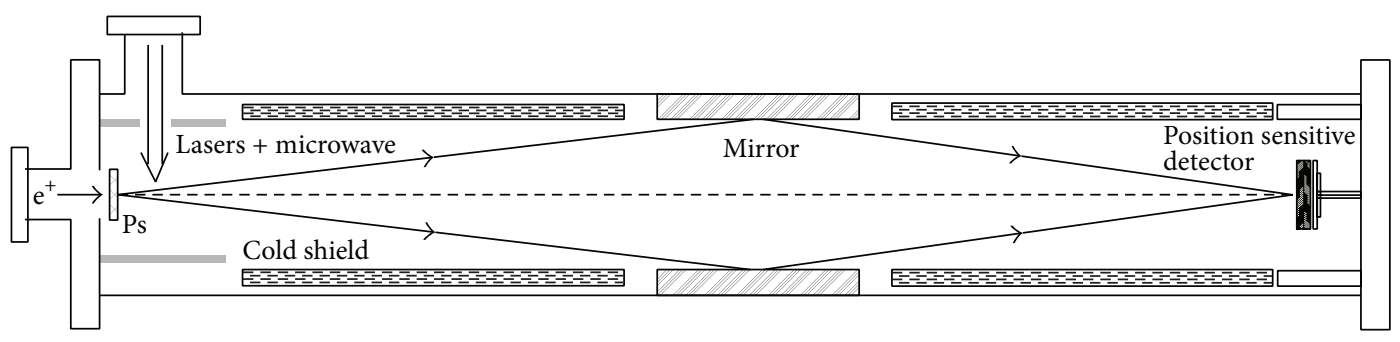

FIGURE 10: A schematic representation of a Mills-Leventhal type of Ps free fall experiment. A real experiment will undoubtedly be significantly different from this illustration, which is intended only to highlight some of the different steps involved. Of distinct practical concern will be the need to keep the apparatus at low temperatures to mitigate effects of black body radiation, as well as minimizing the Ps speed, which will determine the length of the flight path and hence the experiment.

electromagnetic fields dominating their motion, excitation to states with very large dipole moments brings us back to the original problem of extraneous field effects. The situation is considerably less dire when dealing with electric dipoles and, to a much lesser extent, magnetic dipoles, since, in this case, only field gradients give rise to forces; nevertheless, in an experiment designed to probe the weak force of gravity with Rydberg atoms, forces due to stray fields must be taken into account. Magnetic dipoles are less important in this regard. Ordinarily, atoms in weak magnetic fields will experience linear Zeeman shifts, but, in the case of ortho-Ps, there is no permanent magnetic dipole moment because the electron and positron contributions cancel exactly. Thus, only diamagnetic interactions or induced dipole moments have to be taken into account, which we expect to be negligible for realistic experimental conditions.

When an atom is placed in an external electric field of strength $F$ and direction $z$, the field mixes the atom's angular momentum states. To first-order, the state $|n, l, m\rangle$ is mixed with states of adjacent $l$ but the same $n$ and $m$ [59]. The resulting Stark states repel each other, causing them to spread out as the electric field strength is increased, as shown in Figure 11. Following the example of hydrogen, where the first-order Stark shift is analytically calculable, the Schrödinger equation for an atom in an electric field can be written in cylindrical coordinates, where the relevant quantum numbers are $n, m$ and the parabolic quantum numbers are $n_{1}$ and $n_{2}$, which together satisfy the condition $n=n_{1}+n_{2}+|m|+1$.

The Stark states in a given $|n, m\rangle$ manifold are described by the index $k=n_{1}-n_{2}$, where $k$ has values in the range from $k_{\min }=-(n-|m|-1)$ up to $k_{\max }=n-|m|-1$ (with $\Delta k=2)$. The first-order Stark shift in Ps is given by $E_{S}=$ $-\mu \times F$, where the electric dipole moment has magnitude $|\mu|=$ $(3 / 2) n|k| a_{\mathrm{Ps}}$ (where the Ps Bohr radius $a_{\mathrm{Ps}}$ is (almost) twice that of hydrogen, i.e., $2 a_{0}$ ). For a high- $n$ Rydberg state with low angular momentum, for example, the 30d state with $m=$ 2 , the value of the electric dipole moment can be very large. The Stark state with $k_{\max }=27$ has an electric dipole moment of $2430 e a_{0}$. This large electric dipole moment arises because within this $n$-state there are many degenerate angular momentum states with the same value of $m$ that are coupled by the electric field. While this is extremely useful for atomic control $[56,60,61]$, it presents a significant problem

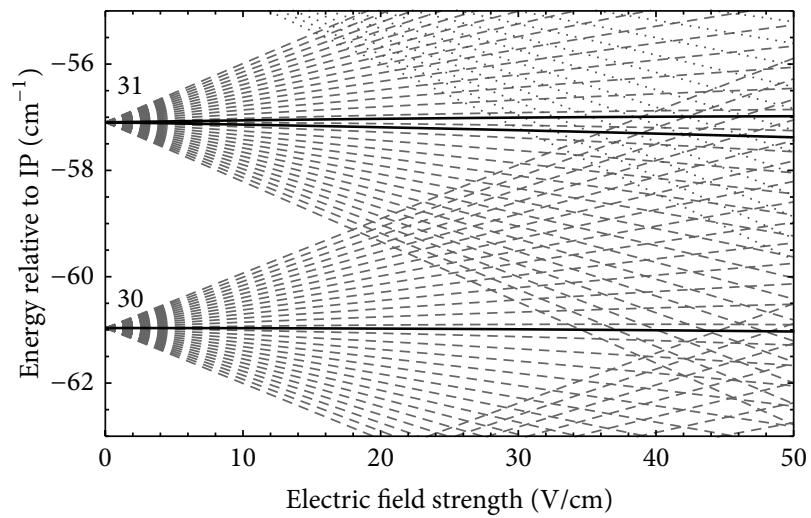

FIGURE 11: Stark states of $n=30$ and 31 states of Ps, with $m=2$ (grey dashed) and $m=29$ (black). In the $n=30$ level, the $m=29$ state is a circular state and experiences no first-order Stark shift and only a very weak second-order shift, as explained in the text.

for gravity measurements, since the electric field gradient experienced by a Ps atom in this state which would result in a force equal to that of "normal" gravity $\left(\sim 2 \times 10^{-29} \mathrm{~N}\right)$ is only $\sim 10^{-3} \mathrm{~V} / \mathrm{m}^{2}$. Although this is by no means insignificant, it does compare favorably with the $\sim 5 \times 10^{-11} \mathrm{~V} / \mathrm{m}$ electric field that would apply a $g$-like force to a bare positron (or electron).

States with the maximum absolute values for the orbital and magnetic quantum numbers for a given $n$, the so-called circular states, experience no first-order Stark shift. For these states, $m=|n|-1$, meaning that there is only one Stark state associated with this value of $m$, which has $k=0$, and, thus, to first-order, no electric dipole moment (see Figure 11). The explanation for this is that, within a given $n$-manifold, the circular states have unique values of $m$, and thus are not coupled to any other degenerate angular momentum states. In the classical limit, these states correspond to circular orbits, in which the average $z$-position of the electron is zero, resulting in no electric dipole moment, unlike the lower angular momentum Rydberg states where, in the classical limit, the electronic orbit is highly anisotropic, with the electron having a large average displacement from the atomic core. Although there is no atomic core or nucleus in the case of Ps, the wave function is nevertheless hydrogenic, and the same arguments apply. The circular states do experience a second-order Stark 


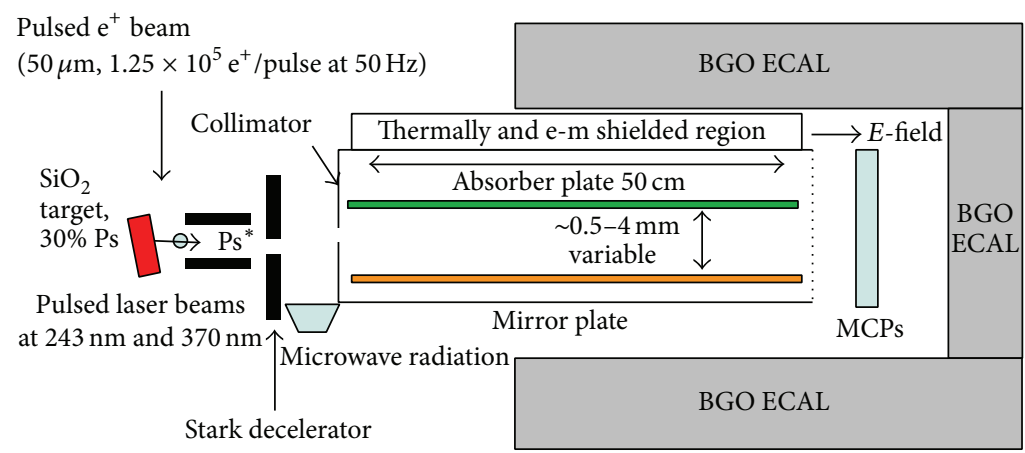

FIgURE 12: Possible scheme for the observation of the gravitational quantum states of positronium.

shift, from coupling of adjacent $n$-states; however, this shift is extremely weak. It should, therefore, be possible to produce Ps states with high angular momentum and minimize the effects of stray fields while simultaneously extending the lifetimes to useful levels. However, any manipulation techniques that rely on large dipole moments will obviously have to be performed after the optical excitation to the relevant $n$ states, but before transferring the atoms to states with high angular momentum.

Performing a gravity measurement on any system containing antimatter is clearly very challenging, and many of the potential obstacles are currently being investigated. The ability to produce controllable beams of Ps atoms may also open the door to other types of experiments, such as interferometry $[62,63]$, which could provide an alternative route to an antimatter gravity measurement.

\section{Can We Observe Gravitational Quantum States of Positronium?}

Positronium is about 1000 times lighter than a neutron or antihydrogen. Therefore, the expected height of the gravitational quantum state is 100 larger corresponding to a macroscopic size of $H_{1}=1.3 \mathrm{~mm}$ while the energy is 10 times smaller, $E_{1}=0.13 \mathrm{peV}$ (see (1)-(2)). The observation time to resolve a quantum gravitational state can be estimated using the Heisenberg uncertainty principle to be of the order of $\hbar / E_{1} \simeq 4.5 \mathrm{~ms}$. This value is much larger than the longlived triplet positronium lifetime in the ground state which is $142 \mathrm{~ns}$ (the Ps singlet state only lives $125 \mathrm{ps}$ and thus, in the following, we will only consider the triplet state and refer to it as Ps). Hence, as for the case of a measurement of the gravitational free fall of Ps described in the previous section, the Ps lifetime must be increased by excitation to a higher level. A possible scheme to observe the Ps gravitational quantum states could employ the flow-through technique used for the first observation of this effect with neutrons (see Figure 12). Greater details of the proposed experimental setup and technique are described in a dedicated contribution to this workshop [64]. Here, we describe the main idea.

Positronium is formed by implanting $\mathrm{keV}$ positrons from a remoderated pulsed slow positron beam in a positronpositronium converter. To observe the quantum mechanical behavior of Ps in the gravitational field, its vertical velocity should be of the same order of the gravitational energy levels and thus $v_{y}<0.15 \mathrm{~m} / \mathrm{s}$. Furthermore, to resolve the quantum state, the Ps atom has to interact long enough with the slit and, therefore, it has to be laser excited to a Rydberg state with $n>30$ and maximum $l$ quantum number (see previous section). To keep a reasonable size of the experimental setup (i.e., a slit size of the order of $0.5 \mathrm{~m}$ ) and minimize the number of detectors, the velocities in the horizontal plane should be smaller than $v_{x, z}<100 \mathrm{~m} / \mathrm{s}$. Similar to neutrons a collimator could be used to select the velocity components $v_{x}, v_{y}$ of the positronium distribution. However, since no reliable thermal cold source of positronium exists, the velocity component perpendicular to the surface $v_{z}$ has to be lowered by some other means. Relying on the fact that atoms in Rydberg states have a large dipole moment, Stark deceleration can be used for this purpose. This method has been demonstrated for different atomic species (including hydrogen) [61] and molecules [65]. Atoms in Rydberg states have large dipole moments; thus, electric field gradients can be used to manipulate them. The acceleration/deceleration $a$ imparted to the Rydberg atoms is given by

$$
a=76 \nabla F \frac{1}{m} n k
$$

where $\nabla F$ is the gradient of the electric field in $\mathrm{V} \mathrm{cm}{ }^{-2}, m$ is the mass of the decelerated particles in atomic units, and $n$ and $k$ are the Stark state quantum numbers. H atoms in $n=25$ and an initial velocity of $700 \mathrm{~m} / \mathrm{s}$ can be brought at rest in $3 \mathrm{~mm}[61]$.

As Ps is 1000 times lighter, decelerations exceeding $10^{9} \mathrm{~m} / \mathrm{s}$ could be realized and therefore the vertical velocity of Ps emitted from thin silica films with initial velocities of the order of $10^{5} \mathrm{~m} / \mathrm{s}[66,67]$ could be reduced to below $100 \mathrm{~m} / \mathrm{s}$. Since one is interested only in decelerating the distribution that is almost perpendicular to the surface of the Ps target, one can expect for those atoms an efficiency close to $100 \%$. This is confirmed by preliminary simulations. The collimator will be placed after the deceleration stage and the microwave region where circularly polarized radiation will spin up the Ps to the maximum $l$ so that kicks to the momentum imparted to the atoms in the vertical direction during these processes will be accounted for. 
The fraction of atoms with $v_{y}<0.15, v_{x}, v_{z}<100 \mathrm{~m} / \mathrm{s}$ is estimated to be of the order of $2 \times 10^{-9}$. After the collimator, the Ps will fly through the slit made of a mirror and the absorber. If the distance between them is smaller than the first expected gravitational state (i.e $<1 \mathrm{~mm}$ ), this will not be transparent and, therefore, no signal will be detected above the expected background in the detectors. If the width of the slit is increased to a value lying between the first and the second gravitational state (i.e. $<2 \mathrm{~mm}$ ), the Ps wave function can propagate and a signal is expected to be detected via field-ionization and subsequent detection with MCPs. This quantum jump would provide the unambiguous indication of the observation of a quantum gravitational state of positronium.

As a mirror for Ps, it was proposed to exploit a gradient of magnetic field created using wires arranged parallel to each other with a constant current to create a uniform gradient of the magnetic field. Only the Ps triplet atoms with $m=0$ have a nonzero net magnetic moment. For the $m= \pm 1$, the electron and the positron magnetic moments cancel and therefore those are insensitive to the magnetic field. Therefore, only one third of the initial population will be reflected. To equate the $E_{y}=0.1 \mathrm{peV}$ a field of few $\mathrm{mG}$ at the wire surface will be sufficient.

Because of the large spacial size of gravitational quantum states and the very large characteristic length of the mirror needed to form the gravitational states that is much larger than a characteristic interwire distance, we expect that the very weak magnetic gradient will not perturb the gravitational states. The strict theoretical analysis of this clearly mathematically defined problem is ongoing. A matter mirror could also be considered. Due to the large spacial size of the gravitational quantum state, the surface potential is expected to be very sharp and therefore results in efficient quantum reflection (see Section 3). In both cases (magnetic or material mirror), we expect to have effectively (quasiclassically) only a few collisions with the surface. Nevertheless, the transitions rates due to quenching and ionization caused by the electric or magnetic fields have to be calculated. The absorber as for the neutrons is a rough surface on which the impinging Ps will mix its velocity components and therefore be lost.

With such a scheme assuming a monoenergetic slow positron beam flux of $9 \times 10^{8} \mathrm{e}^{+} / \mathrm{s}$ (this being the highest intensity reported so far reached at the FMR II NEMOPUC source in Munich [68]), an event rate of 0.8 events/day with a background 0.05 events/day might be achievable with a realistic extrapolation of current technologies. Possible losses due to spurious effects like stray electric or magnetic fields or black body radiation seem to be negligible but as for the case of a free gravity fall further calculations and preliminary experiments should be done to confirm this assumption and that all the required efficiencies (e.g., Ps excitation in the $n=33, l=32$ state) can be attained.

Note that the expected height of the gravitational state is related to the gravitational mass $M$ by (2). This means that, for an uncertainty in the determination of $H_{1}$ of $\delta H_{1}$, one can get an accuracy in the determination of $M$ at the level of $\delta M / M=3 \delta H_{1} / H_{1} \sqrt{N}$ where $N$ is the number of detected signals. Assuming an uncertainty of $\delta H_{1}=0.1 \mathrm{~mm}$ which is mainly determined by the finite source size, the value of $\delta M / M$ can be determined to be at a level of $3 \%$ in three months. This precision is comparable to the one that is aimed for by the antihydrogen experiments at CERN [69-71]. Therefore, observation of Ps gravitational quantum states offers a complementary approach to test the effect of gravity on a pure leptonic system. Most of the techniques required for such an experiment are under development for the ongoing free gravity fall experiment of Ps (see Section 6) and Rydberg Ps deceleration experiments are being considered at ETH Zurich where Professor B. Brown's (Marquette University) buffer gas trap is being commissioned. The advantage of using gravitational quantum states is that unpredicted perturbations of the Ps atoms will not result in a systematic effect for the experiment but will only affect the signal rate. Therefore, as for the case of antihydrogen, this approach seems promising to provide a much higher accuracy than a free fall experiment.

\section{Conclusion}

In this contribution, we have reported the progress of ongoing experiments to measure gravitational free fall of antimatter. The GBAR experiment will produce antihydrogen atoms in the ultracold regime where quantum reflection from surfaces takes place. Quantum reflection will allow the observation of gravitational quantum states of antimatter which promise to lead to a very sensitive probe of the effect of gravity on antiatoms ( 2 orders of magnitude improvement compared to the free fall experiments).

The techniques developed in experiments designed to produce a cold beam of Ps for a free fall measurement will also eventually find application in creating ultracold Ps atoms, as required for observing gravitational quantum states. They will also enable a wide variety of other experimental areas, such as precision spectroscopy.

Antimatter atoms in gravitational quantum states also provide a unique opportunity to constrain experimentally extra short-range forces between the mirror and the antiatom with about the same sensitivity as we do for normal matter [72].

\section{Conflict of Interests}

The authors declare that there is no conflict of interests regarding the publication of this paper.

\section{Acknowledgments}

The authors wish to thank the GRANIT collaboration and the GBAR collaboration (http://gbar.in2p3.fr/) for providing excellent possibilities for discussions and exchange. In addition to the involved institutes and laboratories, preparatory work for GBAR has been funded by the "Conseil Général de l'Essonne," by the "Agence Nationale de la Recherche" in France and by the LABEX P2IO. P. Crivelli acknowledges the support by the Swiss National Science Foundation (Grant PZ00P2_132059) and ETH Zurich (Grant ETH-47-12-1). D. B. 
Cassidy and T. E. Wall gratefully acknowledge funding from the EPSRC (EP/K028774/1), the Leverhulme Trust (RPG2013-055), and the ERC (CIG 630119). This work reviews contributions made at the GRANIT 2014 workshop on prospects for the observation of the free fall and gravitational quantum states of antimatter.

\section{References}

[1] The ALPHA Collaboration and A. E. Charman, "Description and first application of a new technique to measure the gravitational mass of antihydrogen," Nature Communications, vol. 4, article 1785, 2013.

[2] AEGIS Collaboration and M. G. Giammarchi, "AEGIS at CERN: measuring antihydrogen fall," Few-Body Systems, vol. 54, no. 56, pp. 779-782, 2013.

[3] P. Pérez and Y. Sacquin, "The GBAR experiment: gravitational behaviour of antihydrogen at rest," Classical and Quantum Gravity, vol. 29, no. 18, Article ID 184008, 2012.

[4] K. Kirch and K. S. Khaw, "Testing antimatter gravity with muonium," International Journal of Modern Physics: Conference Series, vol. 30, Article ID 1460258, 8 pages, 2014.

[5] D. B. Cassidy and S. D. Hogan, "Atom control and gravity measurements using Rydberg positronium," International Journal of Modern Physics: Conference Series, vol. 30, Article ID 1460259, 9 pages, 2014.

[6] M. M. Nieto and T. Goldman, "The arguments against "antigravity" and the gravitational acceleration of antimatter," Physics Report, vol. 205, no. 5, pp. 221-281, 1991.

[7] J. Scherk, "Antigravity: a crazy idea?” Physics Letters: B, vol. 88, no. 3-4, pp. 265-267, 1979.

[8] G. Chardin and J.-M. Rax, "CP violation. A matter of (anti) gravity?” Physics Letters B, vol. 282, no. 1-2, pp. 256-262, 1992.

[9] G. Chardin, "CP violation and antigravity (revisited)," Nuclear Physics, Section A, vol. 558, pp. 477-495, 1993.

[10] G. Chardin, "Motivations for antigravity in general relativity," Hyperfine Interactions, vol. 109, no. 1-4, pp. 83-94, 1997.

[11] V. A. Kostelecky and J. D. Tasson, "Matter-gravity couplings and Lorentz violation,” Physical Review D, vol. 83, no. 1, Article ID 016013, 2011.

[12] M. L. Good, " $K_{2} 0$ and the equivalence principle," Physical Review, vol. 121, pp. 311-313, 1961.

[13] S. Pakvasa, W. A. Simmons, and T. J. Weiler, "Test of equivalence principle for neutrinos and antineutrinos," Physical Review D, vol. 39, no. 6, pp. 1761-1763, 1989.

[14] A. Apostolakis, E. Aslanides, G. Backenstoss et al., "Tests of the equivalence principle with neutral kaons," Physics Letters B: Nuclear, Elementary Particle and High-Energy Physics, vol. 452, no. 3-4, pp. 425-433, 1999.

[15] G. Gabrielse, A. Khabbaz, D. S. Hall, C. Heimann, H. Kalinowsky, and W. Jhe, "Precision mass spectroscopy of the antiproton and proton using simultaneously trapped particles," Physical Review Letters, vol. 82, no. 16, pp. 3198-3201, 1999.

[16] F. C. Witteborn and W. M. Fairbank, "Experimental comparison of the gravitational force on freely falling electrons and metallic electrons," Physical Review Letters, vol. 19, no. 18, pp. 1049-1052, 1967.

[17] M. H. Holzscheiter, "Constraints on gravitational properties of antimatter from cyclotron-frequency measurements," International Journal of Modern Physics: Conference Series, vol. 30, Article ID 1460260, 9 pages, 2014.
[18] Antimatter and Gravity, International Journal of Modern Physics: Conference Series, 2014.

[19] M. Abramowitz and I. A. Stegun, Handbook of Mathematical Functions with Formulas, Graphs, and Mathematical Tables, Wiley, New York, ny, usa, 1972.

[20] L. D. Landau, E. M. Lifshitz, J. B. Sykes, and J. S. Bell, Quantum Mechanics: Non-Relativistic Theory, Royaume-Uni, Oxford, UK, 1965.

[21] V. V. Nesvizhevsky, H. G. Börner, A. K. Petukhov et al., "Quantum states of neutrons in the Earth's gravitational field," Nature, vol. 415, no. 6869, pp. 297-299, 2002.

[22] G. Chardin, P. Grandemange, D. Lunney et al., "Proposal to measure the gravitational behaviour of antihydrogen at rest," Tech. Rep. CERN-SPSC-2011-029. SPSC-P-342, CERN, Geneva, Switzerland, 2011.

[23] J. Walz and T. W. Hänsch, "A proposal to measure antimatter gravity using ultracold antihydrogen atoms," General Relativity and Gravitation, vol. 36, no. 3, pp. 561-570, 2004.

[24] J. P. Merrison, H. Bluhme, J. Chevallier et al., "Hydrogen formation by proton impact on positronium," Physical Review Letters, vol. 78, no. 14, pp. 2728-2731, 1997.

[25] H. R. J. Walters and C. Starrett, "Positron and positronium scattering," Physica Status Solidi (C), vol. 4, no. 10, pp. 34293436, 2007.

[26] P. Comini and P.-A. Hervieux, " $\bar{H}^{+}$ion production from collisions between antiprotons and excited positronium: cross sections calculations in the framework of the GBAR experiment," New Journal of Physics, vol. 15, no. 9, Article ID 095022, 2013.

[27] L. Liszkay, C. Corbel, P. Pérez et al., "Positronium reemission yield from mesostructured silica films," Applied Physics Letters, vol. 92, no. 6, Article ID 063114, 2008.

[28] D. B. Cassidy, T. H. Hisakado, H. W. K. Tom, and A. P. Mills, "Photoemission of positronium from Si," Physical Review Letters, vol. 107, no. 3, Article ID 033401, 2011.

[29] N. Oshima, T. M. Kojima, M. Niigaki, A. Mohri, K. Komaki, and Y. Yamazaki, "New scheme for positron accumulation in ultrahigh vacuum," Physical Review Letters, vol. 93, no. 19, Article ID 195001, 2004.

[30] P. Grandemange, Piegeage et accumulation de positons issus d'un faisceau pulse produit par un accelerateur pour I'etude de I'interaction gravitationnelle de I'antimatiere [Ph.D. thesis], Universite Paris Sud, Paris, France, 2013.

[31] L. Hilico, J.-P. Karr, A. Douillet, P. Indelicato, S. Wolf, and F. S. Kaler, "Preparing single ultra-cold antihydrogen atoms for free-fall in GBAR," International Journal of Modern Physics: Conference Series, vol. 30, Article ID 1460269, 11 pages, 2014.

[32] H. B. G. Casimir and D. Polder, "Influence of retardation on the London-van der Waals forces," Nature, vol. 158, no. 4022, pp. 787-788, 1946.

[33] H. B. G. Casimir, "On the attraction between two perfectly conducting plates," Proceedings of the Koninklijke Nederlandse Akademie van Wetenschappen, vol. 51, p. 793, 1948.

[34] H. Friedrich, G. Jacoby, and C. G. Meister, "Quantum reflection by Casimir-van der Waals potentials tails," Physical Review A, vol. 65, no. 3 B, Article ID 032902, 2002.

[35] A. Lambrecht, P. A. Maia Neto, and S. Reynaud, "The Casimir effect within scattering theory," New Journal of Physics, vol. 8, article no. 243, 2006.

[36] R. Messina, D. A. R. Dalvit, P. A. M. Neto, A. Lambrecht, and S. Reynaud, "Dispersive interactions between atoms and 
nonplanar surfaces," Physical Review A, vol. 80, no. 2, Article ID 022119, 2009.

[37] G. Dufour, A. Gerardin, R. Guérout et al., "Quantum reflection of antihydrogen from the Casimir potential above matter slabs," Physical Review A, vol. 87, no. 1, Article ID 012901, 2013.

[38] M. V. Berry and K. E. Mount, "Semiclassical approximations in wave mechanics," Reports on Progress in Physics, vol. 35, no. 1, article no. 306, pp. 315-397, 1972.

[39] A. Y. Voronin, P. Froelich, and B. Zygelman, "Interaction of ultracold antihydrogen with a conducting wall," Physical Review A, vol. 72, no. 6, Article ID 062903, 2005.

[40] G. Dufour, R. Guerout, A. Lambrecht, V. V. Nesvizhevsky, S. Reynaud, and A. Y. Voronin, "Quantum reflection of antihydrogen from nanoporous media," Physical Review A, vol. 87, no. 2, Article ID 022506, 2013.

[41] G. Dufour, P. Debu, A. Lambrecht, V. V. Nesvizhevsky, S. Reynaud, and A. Y. Voronin, "Shaping the distribution of vertical velocities of antihydrogen in GBAR," European Physical Journal C, vol. 74, no. 1, article 2731, 2014.

[42] V. V. Nesvizhevsky, H. Börner, A. M. Gagarski et al., "Search for quantum states of the neutron in a gravitational field: gravitational levels," Nuclear Instruments and Methods in Physics Research, Section A: Accelerators, Spectrometers, Detectors and Associated Equipment, vol. 440, no. 3, pp. 754-759, 2000.

[43] V. V. Nesvizhevsky, H. G. Börner, A. M. Gagarski et al., "Measurement of quantum states of neutrons in the Earth's gravitational field," Physical Review D, vol. 68, no. 10, Article ID 102002, 2003.

[44] V. V. Nesvizhevsky, A. K. Petukhov, H. G. Börner et al., "Study of the neutron quantum states in the gravity field," European Physical Journal C, vol. 40, no. 4, pp. 479-491, 2005.

[45] A. Y. Voronin, H. Abele, S. Baeßler et al., "Quantum motion of a neutron in a waveguide in the gravitational field," Physical Review D, vol. 73, no. 4, Article ID 044029, 2006.

[46] A. Y. Voronin, P. Froelich, and V. V. Nesvizhevsky, "Gravitational quantum states of Antihydrogen," Physical Review A: Atomic, Molecular, and Optical Physics, vol. 83, no. 3, Article ID 032903, 2011.

[47] W. E. Lamb, "Fine structure of the hydrogen atom. III," Physical Review, vol. 85, no. 2, pp. 259-276, 1952.

[48] L. P. Gor'kov and I. E. Dzyaloshinskii, "Contribution to the theory of the Mott exciton in a strong magnetic field," Soviet Physics-JETP, vol. 26, pp. 449-453, 1968.

[49] Y. E. Lozovik and S. Y. Volkov, "Hydrogen atom moving across a magnetic field," Physical Review A: Atomic, Molecular, and Optical Physics, vol. 70, no. 2, Article ID 023410, 2004.

[50] A. Y. Voronin and P. Froelich, "Quantum reflection of ultracold antihydrogen from a solid surface," Journal of Physics B: Atomic, Molecular and Optical Physics, vol. 38, no. 18, pp. L301-L308, 2005.

[51] M. Kreuz, V. V. Nesvizhevsky, P. Schmidt-Wellenburg et al., "A method to measure the resonance transitions between the gravitationally bound quantum states of neutrons in the GRANIT spectrometer," Nuclear Instruments and Methods in Physics Research A: Accelerators, Spectrometers, Detectors and Associated Equipment, vol. 611, no. 2-3, pp. 326-330, 2009.

[52] A. P. Mills Jr., "Positron moderation and remoderation techniques for producing cold positron and positronium sources," Hyperfine Interactions, vol. 44, no. 1-4, pp. 105-123, 1989.

[53] H. A. Bethe and E. E. Salpeter, Quantum. Mechanics of One-And Two-Electron Atoms, Dover, New York, NY, USA, 1957.
[54] T. F. Gallagher, Rydberg Atoms, Cambridge University Press, Cambridge, UK, 1994.

[55] A. P. Mills Jr. and M. Leventhal, "Can we measure the gravitational free fall of cold Rydberg state positronium?” Nuclear Instruments and Methods in Physics Research, Section B: Beam Interactions with Materials and Atoms, vol. 192, no. 1-2, pp. 102106, 2002.

[56] C. Seiler, S. D. Hogan, H. Schmutz, J. A. Agner, and F. Merkt, "Collisional and radiative processes in adiabatic deceleration, deflection, and off-axis trapping of a Rydberg atom beam," Physical Review Letters, vol. 106, no. 7, Article ID 073003, 2011.

[57] A. C. L. Jones, T. H. Hisakado, H. J. Goldman, H. W. K. Tom, A. P. Mills Jr., and D. B. Cassidy, "Doppler-corrected Balmer spectroscopy of Rydberg positronium," Physical Review A, vol. 90, no. 1, Article ID 012503, 2014.

[58] T. E. Wall, D. B. Cassidy, and S. D. Hogan, This has not yet been demonstrated for positronium; the feasibility of doing so is considered, to be published.

[59] T. F. Gallagher, "Rydberg atoms," Reports on Progress in Physics, vol. 51, no. 2, pp. 143-188, 1988.

[60] E. Vliegen and F. Merkt, "Normal-incidence electrostatic rydberg atom mirror," Physical Review Letters, vol. 97, no. 3, Article ID 033002, 2006.

[61] S. D. Hogan and F. Merkt, "Demonstration of three-dimensional electrostatic trapping of state-selected rydberg atoms," Physical Review Letters, vol. 100, no. 4, Article ID 043001, 2008.

[62] T. J. Phillips, "Antimatter gravity studies with interferometry," Hyperfine Interactions, vol. 109, no. 1-4, pp. 357-365, 1997.

[63] M. K. Oberthaler, "Anti-matter wave interferometry with positronium," Nuclear Instruments and Methods in Physics Research, Section B: Beam Interactions with Materials and Atoms, vol. 192, no. 1-2, pp. 129-134, 2002.

[64] P. Crivelli, V. V. Nesvizhevsky, and A. Y. Voronin, "Can we observe the gravitational quantum states of Positronium?", Advances in High Energy Physics. In press.

[65] S. D. Hogan, C. Seiler, and F. Merkt, "Rydberg-state-enabled deceleration and trapping of cold molecules," Physical Review Letters, vol. 103, no. 12, Article ID 123001, 2009.

[66] D. B. Cassidy, P. Crivelli, T. H. Hisakado et al., "Positronium cooling in porous silica measured via Doppler spectroscopy," Physical Review A: Atomic, Molecular, and Optical Physics, vol. 81, no. 1, Article ID 012715, 2010.

[67] P. Crivelli, U. Gendotti, A. Rubbia, L. Liszkay, P. Pérez, and C. Corbel, "Measurement of the orthopositronium confinement energy in mesoporous thin films," Physical Review A-Atomic, Molecular, and Optical Physics, vol. 81, no. 5, Article ID 052703, 2010.

[68] C. Hugenschmidt, B. Löwe, J. Mayer et al., "Unprecedented intensity of a low-energy positron beam," Nuclear Instruments and Methods in Physics Research A: Accelerators, Spectrometers, Detectors and Associated Equipment, vol. 593, no. 3, pp. 616-618, 2008.

[69] A. Kellerbauer, M. Amoretti, A. S. Belov et al., "Proposed antimatter gravity measurement with an antihydrogen beam," Nuclear Instruments and Methods in Physics Research B: Beam Interactions with Materials and Atoms, vol. 266, no. 3, pp. 351356, 2008.

[70] D. P. van der Werf, “The GBAR experiment," International Journal of Modern Physics: Conference Series, vol. 30, Article ID 1460263, 2014. 
[71] A. I. Zhmoginov, A. E. Charman, R. Shalloo, J. Fajans, and J. S. Wurtele, "Nonlinear dynamics of anti-hydrogen in magnetostatic traps: implications for gravitational measurements," Classical and Quantum Gravity, vol. 30, no. 20, Article ID 205014, 2013.

[72] I. Antoniadis, S. Baessler, M. Büchner et al., "Short-range fundamental forces," Comptes Rendus Physique, vol. 12, no. 8, pp. 755-778, 2011. 

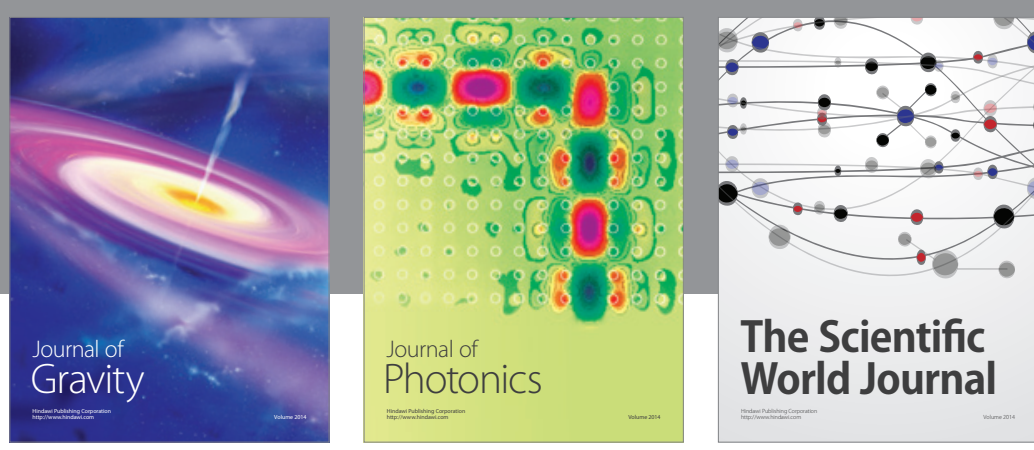

The Scientific World Journal
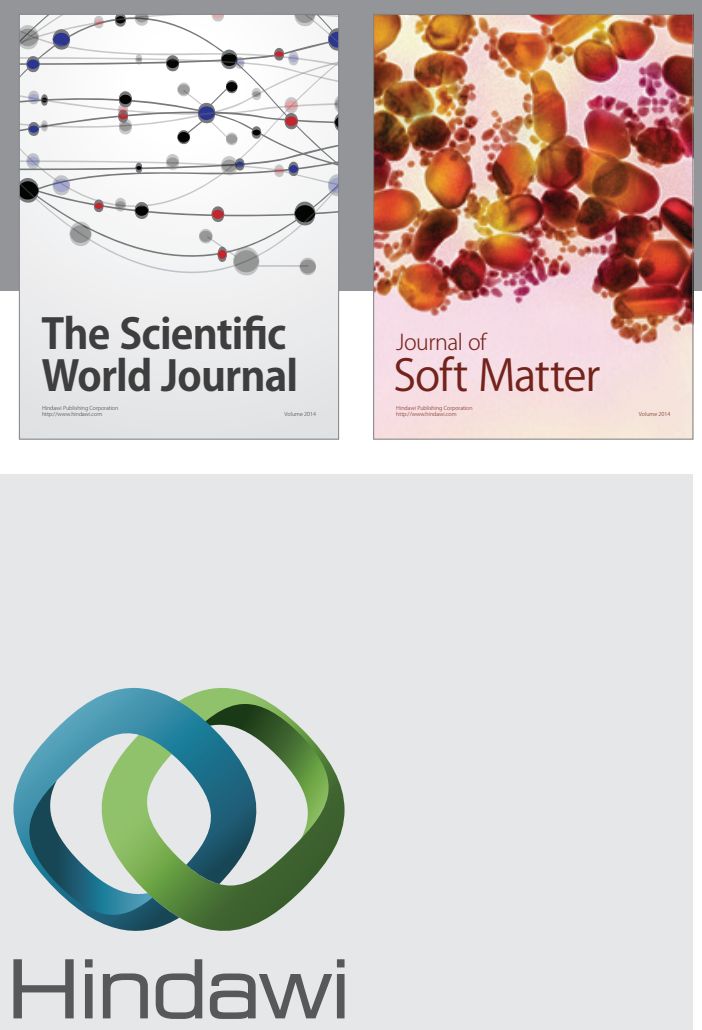

Submit your manuscripts at

http://www.hindawi.com

nternational Journal of

Statistical Mechanics
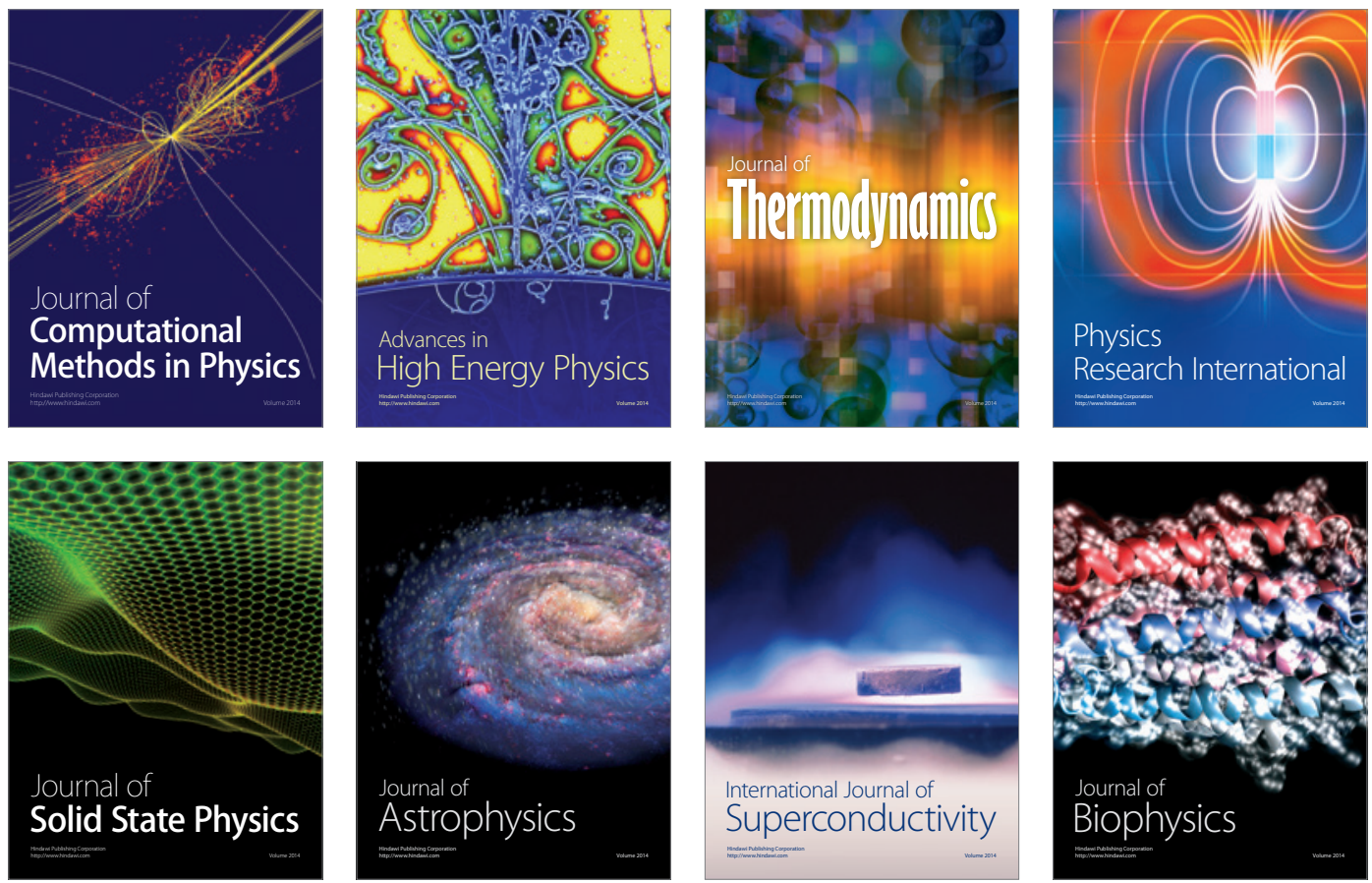
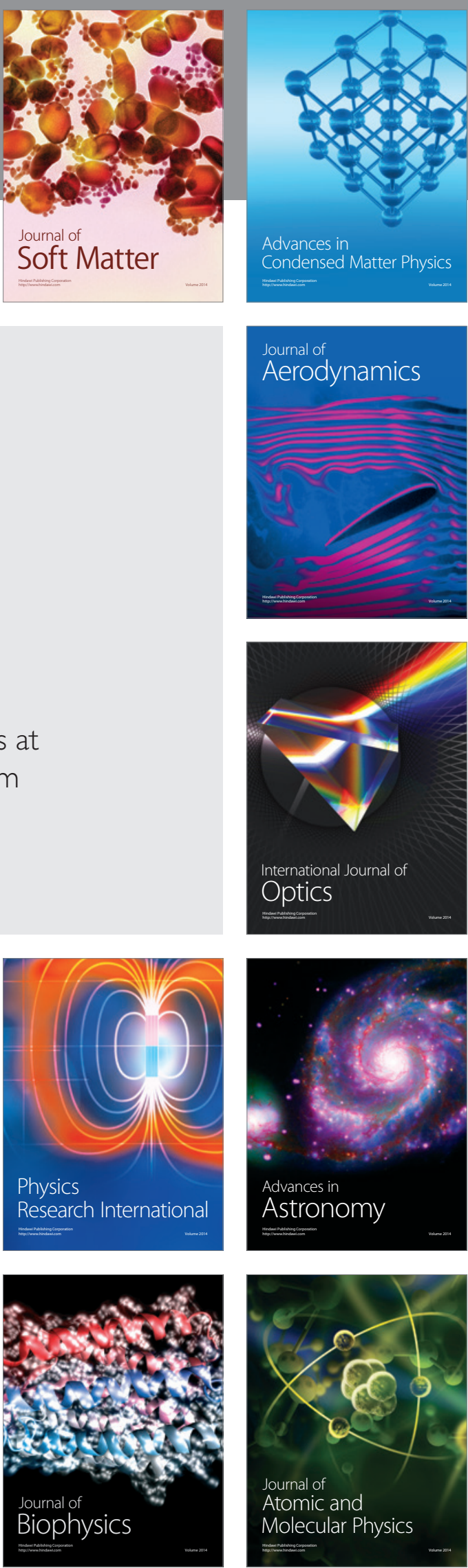